\title{
Numerical Modal Analysis of Vibrations in a Three-Phase Linear Switched Reluctance Actuator
}

\author{
José Salvado, ${ }^{1,2}$ Maria do Rosário Calado, ${ }^{1,3}$ \\ António Espírito Santo, ${ }^{1,3}$ and Anna Guerman ${ }^{3,4}$ \\ ${ }^{1}$ Instituto de Telecomunicações (IT), Calçada Fonte do Lameiro, 6201-001 Covilhã, Portugal \\ ${ }^{2}$ Polytechnic Institute of Castelo Branco, School of Technology, Av. Empresário, S/N, 6000-767 Castelo Branco, Portugal \\ ${ }^{3}$ University of Beira Interior, Faculty of Engineering, Calçada Fonte do Lameiro, 6201-001 Covilhã, Portugal \\ ${ }^{4}$ Center for Mechanical and Aerospace Science and Technologies (C-MAST), Calçada Fonte do Lameiro, 6201-001 Covilhã, Portugal
}

Correspondence should be addressed to José Salvado; josesalvado@ipcb.pt

Received 5 December 2016; Revised 6 February 2017; Accepted 12 March 2017; Published 18 April 2017

Academic Editor: Min-Chie Chiu

Copyright (c) 2017 José Salvado et al. This is an open access article distributed under the Creative Commons Attribution License, which permits unrestricted use, distribution, and reproduction in any medium, provided the original work is properly cited.

\begin{abstract}
This paper addresses the problem of vibrations produced by switched reluctance actuators, focusing on the linear configuration of this type of machines, aiming at its characterization regarding the structural vibrations. The complexity of the mechanical system and the number of parts used put serious restrictions on the effectiveness of analytical approaches. We build the 3D model of the actuator and use finite element method (FEM) to find its natural frequencies. The focus is on frequencies within the range up to nearly $1.2 \mathrm{kHz}$ which is considered relevant, based on preliminary simulations and experiments. Spectral analysis results of audio signals from experimental modal excitation are also shown and discussed. The obtained data support the characterization of the linear actuator regarding the excited modes, its vibration frequencies, and mode shapes, with high potential of excitation due to the regular operation regimes of the machine. The results reveal abundant modes and harmonics and the symmetry characteristics of the actuator, showing that the vibration modes can be excited for different configurations of the actuator. The identification of the most critical modes is of great significance for the actuator's control strategies. This analysis also provides significant information to adopt solutions to reduce the vibrations at the design.
\end{abstract}

\section{Introduction}

The main drawbacks associated with switched reluctance drives (SRD) are the vibrations and the acoustic noise produced in its operation, which are relatively higher than those of the induction machines and permanent magnet synchronous drives. This feature is especially relevant for noise sensitive applications and has received considerable attention over the past two decades. A plethora of studies exists on the analysis of origins and characterization of the mechanical vibrations [1-12], the prediction and mitigation [13-20], and the emitted acoustic noise [11,20-23]. The majority of published works focuses on the rotational configuration of these type of machines, that is, switched reluctance motor (SRM) drives.
When compared to SRM, the linear switched reluctance actuator (LSRA) is a relatively new research and development subject. As a result, the known and published works addressing the vibrations, the force ripple, and the acoustic noise problem for LSRA are relatively scarce [11, 24-28]. Numerous studies identify the primary sources of the acoustic noise as structural vibrations, radiated from the stator, shaft, and bearings and induced by the generated forces. These vibrations vary with the load or the step voltage. Accordingly, several methods have been proposed for either active noise cancellation or new control and optimal driving strategies. For the acoustic noise mitigation, some studies focus on the prediction and characterization of the vibrations. Consequently, several models have been formulated based on the finite element method (FEM), as an alternative to 
analytical or experimental methods, namely, the hammer test method. Among the reasons that explain the use of FEM are the improvements in computational tools and their performance and also the associated capacity to develop complex mechanical models and achieve high accuracy with the results.

The operation of switched reluctance drives is based on the inductance profile of the machine coils, $L_{s}(x)$, which is related to the relative positions of its parts and its dimensions. The operation of a three-phase LSRA is based on the same principle as that of SRM and requires the sequential activation of phase coils $a, b$, and $c$ [29]. If the poles of the stator and the teeth of the translator (or rotor) for any phase are at the unaligned position, the inductance reaches its minimum value. When a phase is activated, the electric current flowing through the coil induces an electromagnetic force that causes the moving parts to move towards the maximum inductance position (minimum magnetic reluctance), that is, to the aligned position. If the movement continues the inductance decreases with the misalignment, due to the increase in the relative displacement towards the minimum inductance. Assuming ideal conditions, the excitation current in the phase coil produces an electromagnetic motoring force that is expressed as [29] (pp. 22)

$$
F_{e m}=\frac{\left(I_{m}\right)^{2}}{2} \cdot \frac{d L_{s}(x)}{d x},
$$

where $I_{m}$ is the peak value of the coil excitation current, $i_{s}$. At the same time, due to the continuous displacement, regenerative forces, $F_{e r}$, occur in the regions adjacent to the full alignment position, such that $F_{e r}=-F_{e m}$, which are related to the negative variation of the inductance in phase coils $b$ and $c$, respectively. In general, the regenerative forces superimpose to the motoring force. Repeated activation of the phases in sequence $a b c$ moves the translator forward and backwards when the sequence $a c b$ is activated. The smoothness of the displacement of the translator depends on the switching positions of the phases, on the duty cycle of the excitation, on the electronics converter topologies, and on the control mode strategies.

In real applications, there are imperfections and nonlinearities associated with the inductance profile; besides, the driving currents do not vary linearly either. Moreover, for a given force, the desired excitation current values are achieved by the switching process of the electronics converters and the control strategies, usually modeled by a pulse width modulation signal. The switching regime causes variations to the current that foster ripple in the produced forces. Thus, the nature of the forces generated during the LSRA operation is susceptible to generating vibration whose magnitude can be amplified, especially in the vicinity of the natural frequencies of structural vibrations. The natural modes, the resonant frequencies, and the characteristics of the induced acoustic noise influence or restrict the use of machines, with direct consequences for human well-being and health [30]. On the other hand, the mechanical vibrations are particularly relevant when precise movement is a requirement and can compromise the application of the actuator.
The sources of vibrations and the acoustic noise in LSRA are primarily the same as for the rotational configuration, due to their common modes of operation. However, the characteristics of the vibrations in electromechanical linear actuators evidence some unique features. Firstly, the natural modes depend on the structural characteristics of the actuator, which vary according to the relative position of the moving parts. Secondly, while vibrations are usually periodic in rotational configuration, in linear actuators the vibrations are periodic only if the excitation forces and the translation movements are periodic. This phenomenon occurs due to linear displacements in this type of machine, rather than angular movement in the rotational configuration. Also, the vibrations tend to be localized, and the associated displacements depend on the position and the structural configuration. Moreover, the finite length of the machine parts influences the propagation of the mechanical waves along the structure. The reflections at both ends interfere along the actuator structure, forming a stationary wave.

The vibrations and the emitted acoustic noise are directly connected with structural aspects of the actuator and its characteristics related to the properties and the dimensions of the materials used. These characteristics dictate the mechanical vibration behavior of the machine and are not an easy issue to deal with from the analytical point of view, in part due to the difficulties encountered in modeling the mechanical structures. Among the structural differences between the rotational and linear configurations of SRD, one can mention the finite length of both the stator and the translator of the latter. On the one hand, the complexity of the mechanical model demands attention to the distribution of masses according to the different positions of the translator. On the contrary, the elastic model denotes results in an increase in complexity, and its analytical solutions are hard to obtain and might be not accurate enough. For these reasons, it is common to use computational simulation tools based on the finite element method (FEM) to model these structures.

The FEM is essentially a discretization technique and approximation method for modeling the distributed parameter systems that correspond to its decomposition into several finite elements or building blocks, equivalent to a system discretization technique. When applied to linear systems, the finite element analysis (FEA) allows finding the approximate solutions to the differential equations that describe the physical model $[31,32]$.

This paper presents a simulation study based on the finite element method to obtain the structural vibration modes and frequencies of an LSRA. The focus relies on frequencies within the range up to nearly $1.2 \mathrm{kHz}$. This band includes the vibration modes considered most relevant and critical to the actuator operation, according to the operation modes as forces profile of the actuator $[17,33]$. Moreover, it complements preliminary results of simulations and experiments for frequencies up to $300 \mathrm{~Hz}$ [26]. The primary objective of this work is to collect data and establish a framework to characterize the linear actuator fully, regarding the vibrations modes induced due to the regular operation of the machine. Due to the lack of a priori knowledge regarding vibrations, we build a $3 \mathrm{D}$ model of the structure and use 


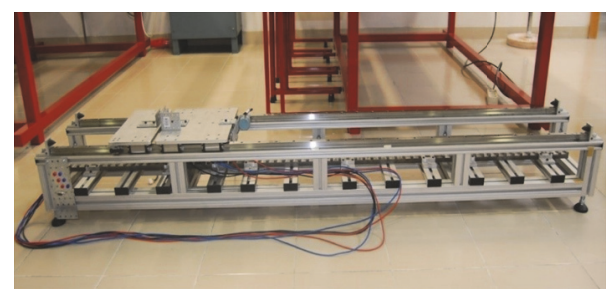

FIGURE 1: General view of the real LSRA considered in this study.

3D FEM simulation software to find the natural vibration frequencies. The data obtained support the characterization of the linear actuator focusing on the excited vibrations and mode shapes. The results reveal abundant modes and harmonics and the symmetry characteristics of the actuator. These findings help to identify the vibration modes that can be excited for different configurations of the actuator, according to the position of its moving parts. The gathered information serves to improve the control of the actuator by avoiding excitation regimes with frequencies near its natural vibration frequencies. It also provides a tool for choosing the configuration of the actuator at the design stage towards the tackling of vibration issues.

The rest of the paper is organized as follows. Section 2 refers to the description and characterization of materials used in the construction of the linear actuator and the threedimensional mechanical model. The analysis of vibrations by the finite element method is applied in Section 3. The results obtained from FEM simulations are presented and discussed in Section 4 and verified by experimental results in Section 5. Finally, Section 6 addresses the key findings and the conclusions.

\section{Characterization of the LSRA and Its 3D Mechanical Model}

The LSRA that supports this study has been developed and built aiming to analyze and validate the application of variable switched reluctance technology for high precision applications [31, 32]. Figure 1 presents its structural overview.

The LSRA of Figure 1 has a total mass of $57 \mathrm{~kg}$ and contains around 180 parts bonded and screwed. Its most relevant physical dimensions (in $\mathrm{mm}$ ) are total length of 2000 , width of 500, and height of 250 . The LSRA parts are mainly profiled frames of aluminum alloy 6060-O (AlMgSi 0.5F25) manufactured by Bosch Rexroth [33], except those directly related to the magnetic circuit of the actuator which are of carbon magnetic steel. The primary section of the actuator includes three subassemblies that correspond to each one of its phases and includes aluminum parts for fastening and support and steel parts in the magnetic circuit. Each subassembly includes the coil, encompassing 1092 turns of AWG-20 copper wire and the respective coil core plates and the poles of each phase of the primary. Both the core coil and the phase poles are unique nonlaminated pieces of carbon steel S235-JR, according to EN 10025 (ST37-2 under DIN 17100) with $10 \mathrm{~mm}$ thickness. The secondary is also a unique nonlaminated piece of S235-JR steel with $20 \mathrm{~mm}$ thickness (maximum). The top face has regularly distributed longitudinal cavities with $20 \mathrm{~mm}$ of length and $5 \mathrm{~mm}$ of depth interleaved with $10 \mathrm{~mm}$ wide saliences, forming the teeth. The linear ball guide rails are chromium-plated stainless steel bars; the precision runner blocks associated with the platforms and the locking parts are of stainless steel and composite materials.

To use the FEM to estimate the natural frequencies and the respective mode shapes, we draw the $3 \mathrm{D}$ mechanical model of the main subassemblies that constitute the LSRA using SolidWorks 2013, namely, the base and platforms subassemblies, as depicted in Figures 2(a) and 2(b), respectively. The first includes the bar that constitutes the secondary of the magnetic circuit, and the later incorporates the parts of the primary, namely, the coils and teeth for each coil and the auxiliary platform. For the final assembly, the platforms subassembly is mounted on top of the base subassembly, thus being capable of performing linear motion across the guide rails due to the fitted runner blocks, thus forming the translator. An example of the magnetic circuit assembly is depicted in Figure 3 showing the relative positioning of the poles of the primary and the teeth at the secondary.

However, both platforms at the translator can move independently. Therefore, it increases the complexity of the problem significantly, due to the high variety of possible mutual positioning of the two platforms. To simplify the problem, we consider the primary and auxiliary as joint pieces behaving as solidary parts. To evaluate the modal frequencies of the LSRA, we consider five different final assembly models, each one regarding a particular position for the platforms assembly. For each position, we assume regular displacements of $375 \mathrm{~mm}$ for its symmetry axis concerning the position aligned with the rightmost end allowed by the guide rail course, which is $1900 \mathrm{~mm}$, as depicted in Figure 4. This configuration corresponds to position P1, as shown in Figure 5(a).

In this position (P1), the outer ridges of the platform plates are aligned with the end face of the rail guide and the displacement of that point is null. Therefore, position P1 is here considered as a reference to the positioning of platforms. For the remaining four cases, we consider regular displacements of $375 \mathrm{~mm}$ on the transversal axis that crosses the geometric center of the platforms. The center is defined by the axis of symmetry of the two platforms subassembly, according to the top plane and the direction orthogonal to that of the (longitudinal) displacement. This axis is orthogonal to the direction of the movement, located at the top plane and is $200 \mathrm{~mm}$ distant from the outer ridges of the platform that is also the ending face of the guide rail.

This way, at position P3, the symmetry axis of the platform is aligned with the longitudinal center axis of the actuator base subassembly as illustrated in Figure 5(b). In positions $\mathrm{P} 2$ and $\mathrm{P} 4$, the displacement courses of the platforms subassembly to the nearest outer ridges are identical. At the leftmost position allowed for the movement of the LSRA platforms, the outer ridges reach alignment to the end of the rail guide that corresponds to position P5. This position 


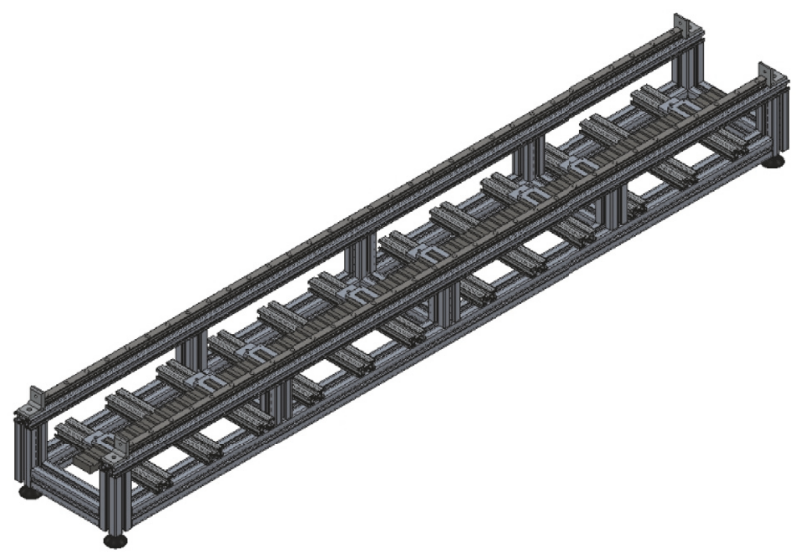

(a)

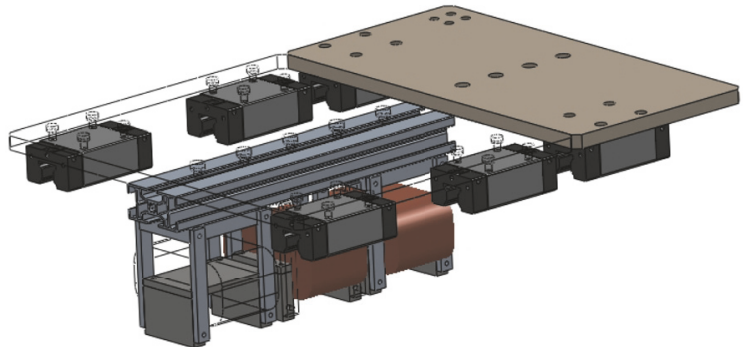

(b)

FIGURE 2: The two main LSRA 3D model assemblies: (a) isometric view of the base parts (longitudinal and transversal bars) and the secondary of magnetic circuit (bar mounted in the middle mounted over transversal bars); (b) perspective view of the platforms subassembly showing the three coils and teeth of the primary of magnetic circuit (some parts shown in wire frame view for reading purposes).

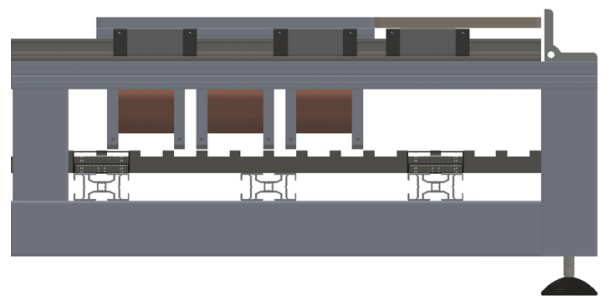

FIgURE 3: Front view of magnetic circuit parts at P1 (poles of phase $b$ aligned with the secondary teeth).

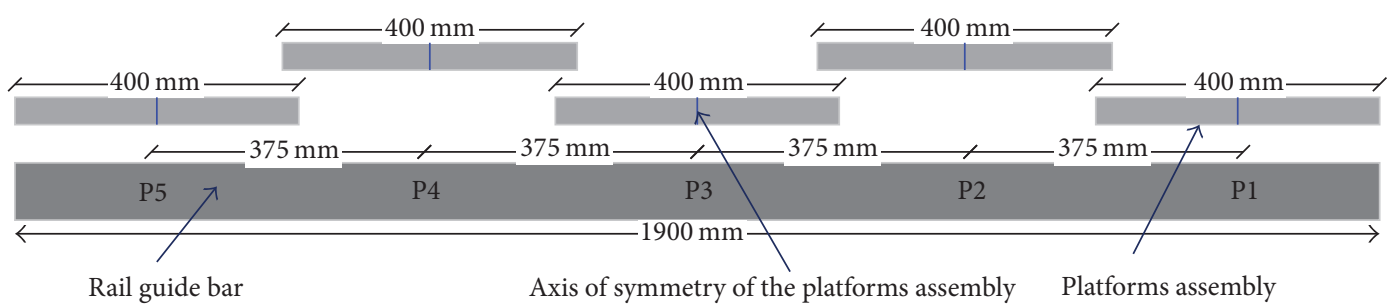

FIGURE 4: Scheme of platforms positions for regular distribution along the top rail guides course.

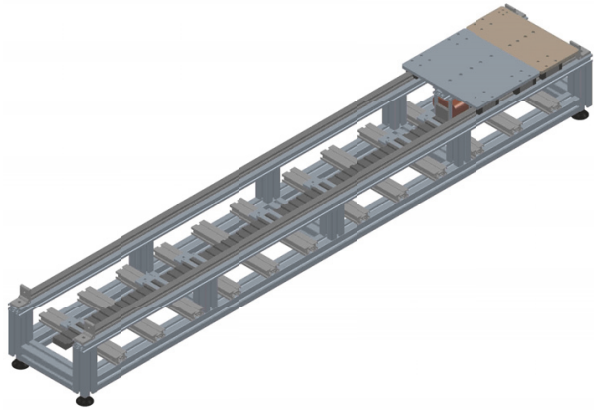

(a)

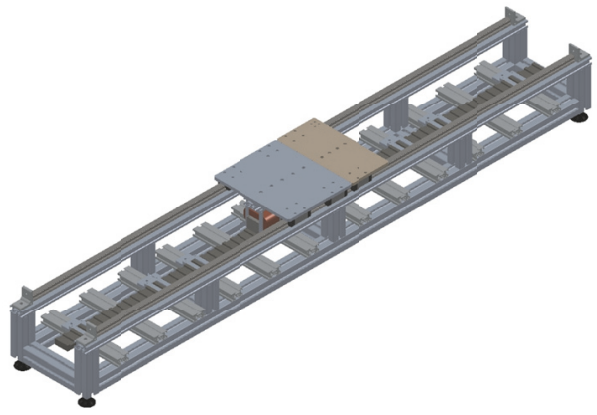

(b)

FIGURE 5: An example of the LSRA configurations regarding the positions of the platforms: (a) at P1, thus aligned at the rightmost end; (b) at $\mathrm{P}$, aligned with the symmetry axis (base and platforms). 
corresponds to a displacement of $1500 \mathrm{~mm}$ to the reference point, that is, P1.

Observing Figures 2 and 3 one can notice that only the base subassembly possesses symmetry as both platform subassemblies are not symmetric. Therefore, the aligned platforms subassembly is also not symmetric. The effect is transference of the center of mass of the actuator for each one of its positions. Consequently, the modal frequencies expected for positions $\mathrm{P} 1$ and $\mathrm{P} 2$ and positions $\mathrm{P} 2-\mathrm{P} 4$ differ from those obtained for their counterpart positions, P5 and P4, respectively.

\section{Principles of Vibrations, Modal Analysis, and FE Simulation}

Most vibrations and acoustic noise problems in machinery are functions of both the forces and the system characteristics, and its modes depend on structural aspects, material properties, and the boundary conditions. The structural complexity and the interconnection of parts often lead to models corresponding to multiple-degrees-of-freedom (MDOF) systems.

3.1. Multiple-Degrees-of-Freedom Systems. The classical structural dynamics theory uses Newton's laws of motion to describe the oscillatory phenomenon. In systems formed by a set of interconnected masses acting through a network of springs and dampers, with viscous damping, submitted to a steady-state sinusoidal excitation upon the action of external forces, the number of independent coordinates defines the number of DOF. The overall system response for MDOF systems, using matrix notation, is given by the motion equation for MDOF damped systems [31] as

$$
[M] \cdot[\ddot{x}]+[C] \cdot[\dot{x}]+[K] \cdot[x]=[F] .
$$

Here $x$ represents the displacements of masses to a reference initial point, $\dot{x}$ the velocities, $\ddot{x}$ the acceleration, and $F$ the external forces, organized as column-wise arrays. The inertia (mass), damping, and stiffness coefficients are represented through square symmetric matrices $[M],[C]$, and $[K]$, respectively, whose dimensions depend on the number of degrees of freedom of the system. The off-diagonal coefficients of matrices $[M],[C]$, and $[K]$, are the coupling terms that depend on the configuration of the system.

3.2. Lagrange's Equations of Motion. An alternative to Newton's laws of motion termed Lagrange's equation consists in describing the motion based on the energy equilibrium in the system [32] (pp. 2.30-2.32), considering the total kinetic $E_{\text {kin }}$ and potential $E_{\text {pot }}$ energies, expressed as

$$
\frac{d}{d t} \frac{\partial\left(E_{\mathrm{pot}}-E_{\mathrm{kin}}\right)}{\partial \dot{x}_{i}}-\frac{\partial\left(E_{\mathrm{pot}}-E_{\mathrm{kin}}\right)}{\partial x_{i}}=0 \begin{aligned}
& \\
& \quad i=1,2, \ldots, n,
\end{aligned}
$$

where $i$ refers to generalized coordinates. The kinetic energy considers all coordinates $i$ associated with the system; the total potential energy is stored in the springs due to compression and displacement $\Delta x$ with respect to an initial position [31] (pp. 294-301). For $n$-DOF systems, Lagrange's equation is

$$
\begin{aligned}
\frac{d}{d t}\left(\frac{\partial E_{\mathrm{kin}}}{\partial \dot{x}_{k}}\right)-\frac{\partial E_{\mathrm{kin}}}{\partial x_{k}}+\frac{\partial E_{\mathrm{pot}}}{\partial x_{k}}+\frac{\partial D_{R}}{\partial \dot{x}_{k}}= & Q_{k} \\
& k=1,2, \ldots, n,
\end{aligned}
$$

where $x_{k}$ is a generalized displacement coordinate; $\dot{x}_{k}$ is the velocity at the generalized coordinate; $D_{R}$ is Rayleigh's dissipation function which represents the energy losses associated with the dumpers; and $Q_{k}$ is relative to the generalized nonconservative forces. For each element of order $k$ the quadratic forms of kinetic and potential energies and Rayleigh's dissipation function can be represented using matrix notation as

$$
\begin{aligned}
E_{\mathrm{kin}} & =\frac{1}{2} \cdot[\dot{x}]^{T} \cdot[M] \cdot[\dot{x}], \\
E_{\mathrm{pot}} & =\frac{1}{2} \cdot[x]^{T} \cdot[K] \cdot[x], \\
D_{R} & =\frac{1}{2} \cdot[\dot{x}]^{T} \cdot[C] \cdot[\dot{x}] .
\end{aligned}
$$

Therefore, considering small displacements with reference to the equilibrium points $\left(x_{k} \approx x_{e k}\right)$, the linearized Lagrange's equation is given by

$$
[M] \cdot\left[\ddot{x}_{k}\right]+[C] \cdot\left[\dot{x}_{k}\right]+[K] \cdot\left[x_{k}\right]=\left[Q_{k}\right] .
$$

It follows that (2) and (8) have a similar representation.

3.3. Theories of Dynamics for Vibrations Analysis in Bars and Plates. The most common approaches to study bars or plates dynamics are that of Euler-Bernoulli and Rayleigh and Timoshenko [32] (pp. 7.19-7.35). Through these methods, the forces acting on the system can be derived from Lagrange's equations although with some limitations; for example, they do not allow for the variation of the bar cross-section when it occurs due to the torsion. Furthermore, due to numerical issues, the accuracy of the Euler-Bernoulli method is considered fair for the first dozen vibration modes [34] but not for higher order modes. Meanwhile, even with these limitations, the first linear approximation analytical method is quite adequate to study the vibrations of uniform bars and find its mode shapes. According to the Euler-Bernoulli approach, applied to orthogonal modes, the equation of motion for a freely vibrating uniform bar with generalized coordinates is a function of displacement and time, expressed as

$$
E I \frac{\partial^{4} w_{i}(x)}{\partial x^{4}}+\rho_{S} S \frac{\partial^{2} w_{i}(x, t)}{\partial t^{2}}=0 .
$$

In (9) $E, I, \rho_{S}$, and $S$ are, respectively, Young's modulus, the second moment of inertia, the density (mass/area), and the cross-sectional area of the bar. Using the Fourier method the deflection function can be expressed in the form

$$
w_{i}(x, t)=\sum_{i} W_{i}(x) \cdot T_{i}(t),
$$


so that $T_{i}(t)$ is a time-harmonic function with solution

$$
T_{i}(t)=\widetilde{A} \cdot \cos \left(\omega_{i} t\right)+\widetilde{B} \cdot \sin \left(\omega_{i} t\right) .
$$

Moreover, the displacement function $W_{i}(x)$ satisfies the expression

$$
\frac{d^{4} W_{i}(x)}{d x^{4}}=\kappa_{i}^{4} \cdot W_{i}(x),
$$

where $\kappa_{i}$ is the flexural wavenumber given by

$$
\kappa_{i}^{4}=\frac{\omega_{i} \cdot \rho_{S} \cdot S}{E \cdot I} \quad i=1,2, \ldots, n .
$$

Considering the existence of time-harmonic motion, the general solution of (11) is a linear combination of trigonometric and hyperbolic functions representing the propagating and evanescent waves [34], respectively, expressed as

$$
\begin{aligned}
W_{i}(x)= & A_{i} \cdot \sin \left(\kappa_{i} x\right)+B_{i} \cdot \cos \left(\kappa_{i} x\right)+C_{i} \\
& \cdot \sinh \left(\kappa_{i} x\right)+D_{i} \cdot \cosh \left(\kappa_{i} x\right) .
\end{aligned}
$$

Here $A_{i}, B_{i}, C_{i}$, and $D_{i}$ are constants that depend on the configuration and the boundary conditions of the bar.

The Euler-Bernoulli method and the above formulation can be used for vibrating beams (or bars) with transversal displacement resulting from external excitation forces, either for single supported bars or for multisupported bars, in this case considering $s-1$, bar segments, if the bar has $s$ supporting points. Hence, considering transversal excitation and normal modes and using (13), one can obtain the frequency of vibration for the $i$ th mode of a bar with length $L_{B}$ and simply supported ends in the form [32] (pp. 7.25)

$$
\omega_{i}=\frac{(i \cdot \pi)^{2}}{L_{B}^{2}} \sqrt{\frac{E \cdot I}{\rho_{S} \cdot S}} \quad i=1,2, \ldots, n .
$$

Alternatively, for higher order modes, one can estimate the frequency of vibration for the $i$ th modes of the same bar using the expression [35]

$$
\omega_{i}=\frac{\pi^{2} \cdot(2 \cdot i-1)^{2}}{\left(2 \cdot L_{B}\right)^{2}} \sqrt{\frac{E \cdot I}{\rho_{S} \cdot S}} \quad i \geq 3 .
$$

However, when used for $i=1$ and $i=2$ its results may have $20 \%$ to $30 \%$ of error [35].

The classical dynamics for vibrations on plates also considers transversal forces applied to the surface of the plate. For rectangular uniform plates, the mode shapes functions are harmonic through the orthogonal coordinates and in the form

$$
w(x, y, t)=w(x) \cdot w(y) \cdot e^{j \omega t}
$$

The frequency of vibrations depends on the particular configuration of the plates and their boundary conditions. Thus, the formulation of the plates' vibrations is rather complex than for the Euler-Bernoulli beam. Hence, the bibliography includes tables specifying the development of the vibration frequencies for plates according to its geometry configuration, for example, in [36] (pp. 41-159).
3.4. Modal Analysis and the Finite Elements Method. The free vibration solution for undamped systems is termed mode and corresponds to a standing wave where all points move at the natural frequency with some phase relation. Thus, each particular eigenvalue, $\lambda_{r}$, corresponds to the undamped natural frequencies and the eigenvectors, $\left[\psi_{r}\right]$, contain information on the mode shapes associated with those frequencies. Because modes have a weighted orthogonality property [31] (pp. 309-310) and the mass, stiffness, and damping matrices are in general Hermitian, it is possible to perform matrix diagonalization and obtain their eigenvectors, therefore simplifying the problem. Therefore, in real systems, one can consider proportional damping as a linear combination of the mass and stiffness matrices [37] and perform the decouple of MDOF systems into modal coordinates. Hence, the equation of motion can be expressed in matrix notation using the form

$$
\left[M_{r}\right] \cdot\left[\ddot{x}_{k}\right]+\left[C_{r}\right] \cdot\left[\dot{x}_{k}\right]+\left[K_{r}\right] \cdot\left[x_{k}\right]=[\psi]^{T} \cdot[Q],
$$

where

$$
\begin{aligned}
{\left[M_{r}\right] } & =[\psi]^{T} \cdot[M] \cdot[\psi], \\
{\left[K_{r}\right] } & =[\psi]^{T} \cdot[K] \cdot[\psi], \\
{\left[C_{r}\right] } & =\alpha\left[M_{r}\right]+\beta\left[K_{r}\right]=[\psi]^{T} \cdot[C] \cdot[\psi] .
\end{aligned}
$$

Each row in (18) corresponds to a single-degree-offreedom (SDOF) system whose equation of motion considers its modal parameters: mass, damping, and stiffness. The undamped natural frequency and the damping coefficient at mode $r$, respectively, are given by

$$
\begin{aligned}
& \omega_{r}=\sqrt{\frac{k_{r}}{m_{r}}}, \\
& c_{r}=-2 \cdot \sigma_{r} \cdot m_{r} .
\end{aligned}
$$

The application of Lagrange's equation to MDOF systems requires the selection of a set of independent coordinates and successive differentiation on each one. Hence, the finite element analysis (FEA) corresponds to decomposition into several building blocks and finds the approximate solutions to the differential equations that describe the model. This procedure is equivalent to a system discretization technique that collects the overall system response as a linear combination of SDOF system responses.

The majority of the LSRA parts model are bars (or beams) either longitudinal or transversal. Regarding the frequency analysis, the bars act as supported-supported or supportedfree bars. The remaining parts of the LSRA include uniform bars with multiple supports along its axis, uniform plates. There are also some nonuniform bars and plates and a few other parts with special configuration. The analysis of bars can be carried out using Euler-Bernoulli or Timoshenko approach; for plates, one can look at the information provided by [36]. However, the multiplicity of parts, their singular geometric configuration and interconnections, and the different materials used increase the complexity of analytical methods. These features suggest the use of FEM simulation tools as a 


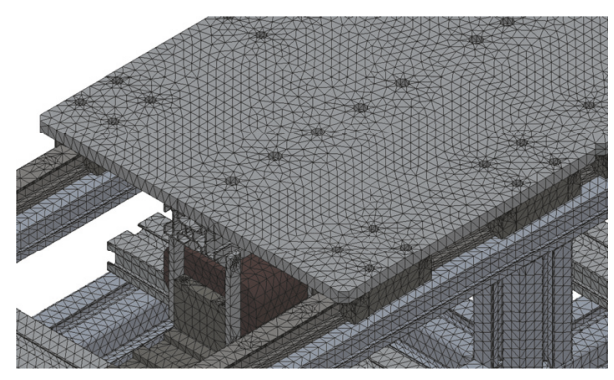

FIGURE 6: Partial detail view of the resultant mesh elements.

more efficient tool for vibration prediction and analysis of the LSRA structure. These are the most significant vibration modes that can be excited during the regular operations of the actuator.

We use the SolidWorks 2013 built simulation tools to perform the FEM frequency analysis study. The global contacts of the actuator parts are considered ideal and set as bonded with no clearance and no penetration allowed among parts. To optimize the simulation results we consider the following:

(i) Curvature based mesh, Jacobian 4 points

(ii) Fine density, with size elements among $1.2 \mathrm{~mm}$ ( $\mathrm{min}$ ) and $12 \mathrm{~mm}$ ( $\max$ )

(iii) 1.6 element growth ratio and, at least, eight elements to describe circles

This configuration preset before FEA leads to a highquality mesh, whose detail view is presented in Figure 6.

The number of elements is approximately $1,221,800$; the total number of nodes is roughly $2,226,200$, and the number of degrees of freedom for $3 \mathrm{D}$ coordinates is $\sim 6,678,700$. The elements having an aspect ratio less than 3 correspond to $28 \%$ and $8 \%$ have an aspect ratio greater than 10 .

\section{Analysis of Vibrations and Discussion of Results}

Based on preliminary experiments [26], the FEA study on the 3D model has been performed for frequencies up to $1.2 \mathrm{kHz}$, which encompasses a set of 80 vibration frequencies and modes per each position of the platforms, one setting a singular configuration for the actuator. Then five scenarios corresponding to positions P1 to P5 are evaluated. Due to the high number of degrees of freedom, the FFEplus solver builtin SolidWorks 2013 is selected, as recommended for DOF greater than 100,000 [36]. This solver uses an iterative integration method to find the solutions and evaluates the individual errors in each iteration; when the latter are small enough, the final settlement is assumed stable. However, when compared to the direct method, the gain in accuracy is also associated with higher computational costs. For example, with the iterative process, the time spent with mesh construction and simulation is around 30 hours on a PC equipped with an Intel Core 2 Duo E7500 CPU @ 2.93 GHz, 6 GB RAM DDR3 @ 1.333 Mbps (36 ns), and running Windows 7 Pro 64 bit operating system. The computational time remains similar for each model considered in this paper.

The results obtained from FEA simulation indicate that the first six frequencies, as expected, are not excited and correspond to rigid body vibration modes. Accordingly, the first significant modal frequency is that of mode 7 , in a set of 74 modes whose frequencies range from nearly $60 \mathrm{~Hz}$ up to approximately $1.2 \mathrm{kHz}$, as indicated in Table 1.

In Table 1 it is noticeable that, for a given mode, the vibration frequencies for all considered positions are rather close to each other. For each configuration, it is also observable that the differences among sequential modal frequencies denote small variations. The average modal frequency interval is $\sim 15 \mathrm{~Hz}$ in each position, but the minimum value can be as small as 1 or $2 \mathrm{~Hz}$ (e.g., modes 41 and 42 or modes 57 and 58, all for P1). Moreover, for adjacent modes at different places, the frequencies can have equal or close values (e.g., mode 24$\mathrm{P} 2$ versus mode $23-\mathrm{P} 3$, and mode $26-\mathrm{P} 3$ versus mode $27-\mathrm{P} 4$ ). That means that a typical vibration frequency can occur due to the excitation of different modes in different positions (see, e.g., mode 36 for all positions).

Due to the complexity of the actuator model, the interconnection among its various parts and subsystems, and the different material used, the information in Table 1 is not sufficient to describe the vibrations. Since each mode corresponds to a dominant vibration associated with any part or subassembly, it is important to look at the shapes of every vibration mode. Moreover, this is also important to evaluate the overall vibration modes of the LSRA, to associate its origins to specific parts or subassemblies, and to establish possible correlations among vibration modes.

Figures 7-9 illustrate the mode shapes associated with the first vibration mode (mode 7), in all the cases examined in the FEA study. These consider a given position and its quasisymmetric counterpart. Note that the images showing the displacements are not true scale. This serves solely to illustrate the most significant displacements for each particular mode. The mode shapes for positions P1 and P5 are antisymmetric and mostly related to torsional displacements of the outer top bars and the guide rails as shown in Figures 7(a) and 7(b). Assuming the view from left to right as a standard reference observation point for both positions, the free end of the machine bends clockwise, in either case. A similar phenomenon occurs for positions P2 and P4, as illustrated in Figures 8(a) and 8(b). However, because the distance from the platforms assembly to the opposite end is lower in this configuration pair, the natural frequencies denote higher values than for positions P1 and P5. Regarding the vibration frequencies and the respective modal shapes and symmetry of displacement, one can observe that the above configurations act as pairs.

The next mode (mode 8) corresponds to vertical bending of the assembly, with higher displacement through the direction $Y Y$ at the ends opposite to the position of the platform. That displacement is more evident at the outside bars for the configuration pair formed by $\mathrm{P} 1$ and $\mathrm{P} 5$, as depicted in Figures 9(a) and 9(b), respectively. Also, as referred to the previous situation, the same occurs for positions $\mathrm{P} 2$ and $\mathrm{P} 4$, as depicted in Figures 10(a) and 10(b). In both configuration pairs, the 
TABLE 1: Vibration modes and modal frequencies for the various configurations of the LSRA.

\begin{tabular}{|c|c|c|c|c|c|}
\hline \multirow{2}{*}{ Mode } & \multicolumn{5}{|c|}{ Modal frequency $[\mathrm{Hz}]$ versus position } \\
\hline & P1 & $\mathrm{P} 2$ & P3 & $\mathrm{P} 4$ & P5 \\
\hline 7 & 67.27 & 76.28 & 83.29 & 74.61 & 66.01 \\
\hline 8 & 114.87 & 114.17 & 106.91 & 118.36 & 109.36 \\
\hline 9 & 142.51 & 133.31 & 107.96 & 138.45 & 137.60 \\
\hline 10 & 175.52 & 177.25 & 160.38 & 171.51 & 169.90 \\
\hline 11 & 178.47 & 179.63 & 196.18 & 182.10 & 182.00 \\
\hline 12 & 183.65 & 185.91 & 197.18 & 182.45 & 192.84 \\
\hline 13 & 192.06 & 201.20 & 226.10 & 198.15 & 200.20 \\
\hline 14 & 229.72 & 224.95 & 230.46 & 238.03 & 225.43 \\
\hline 15 & 231.05 & 233.81 & 232.35 & 246.26 & 226.41 \\
\hline 16 & 236.11 & 259.79 & 245.62 & 252.00 & 255.63 \\
\hline 17 & 274.10 & 265.99 & 267.98 & 259.09 & 262.99 \\
\hline 18 & 274.91 & 279.29 & 273.38 & 270.31 & 263.02 \\
\hline 19 & 281.26 & 283.36 & 292.65 & 273.30 & 275.82 \\
\hline 20 & 303.34 & 301.70 & 302.01 & 307.98 & 291.95 \\
\hline 21 & 310.76 & 321.30 & 315.90 & 323.20 & 308.80 \\
\hline 22 & 312.01 & 324.63 & 327.19 & 329.68 & 310.67 \\
\hline 23 & 319.94 & 328.67 & 340.17 & 352.62 & 317.89 \\
\hline 24 & 349.18 & 340.87 & 362.89 & 362.49 & 342.96 \\
\hline 25 & 364.52 & 370.28 & 378.78 & 366.22 & 359.26 \\
\hline 26 & 391.72 & 385.72 & 389.39 & 386.77 & 381.77 \\
\hline 27 & 393.39 & 393.52 & 393.81 & 389.74 & 393.49 \\
\hline 28 & 401.67 & 412.83 & 407.88 & 393.64 & 406.28 \\
\hline 29 & 408.05 & 420.30 & 424.48 & 421.56 & 417.87 \\
\hline 30 & 445.86 & 444.34 & 426.82 & 439.16 & 446.51 \\
\hline 31 & 460.64 & 458.13 & 435.21 & 441.77 & 452.72 \\
\hline 32 & 464.62 & 459.81 & 456.88 & 457.06 & 460.14 \\
\hline 33 & 482.12 & 487.30 & 485.02 & 475.41 & 480.11 \\
\hline 34 & 507.97 & 496.05 & 493.11 & 484.32 & 506.62 \\
\hline 35 & 517.11 & 527.82 & 515.21 & 520.72 & 512.55 \\
\hline 36 & 535.53 & 533.21 & 533.67 & 532.07 & 535.51 \\
\hline 37 & 561.94 & 536.65 & 538.69 & 536.41 & 560.46 \\
\hline 38 & 568.90 & 551.58 & 548.22 & 563.17 & 562.32 \\
\hline 39 & 571.76 & 567.72 & 569.77 & 567.49 & 568.75 \\
\hline 40 & 609.09 & 609.74 & 612.87 & 600.96 & 591.67 \\
\hline 41 & 613.01 & 610.56 & 614.68 & 604.45 & 600.87 \\
\hline 42 & 615.40 & 624.14 & 619.41 & 608.78 & 613.52 \\
\hline 43 & 621.15 & 626.46 & 643.78 & 620.90 & 619.89 \\
\hline 44 & 634.65 & 639.41 & 645.83 & 630.18 & 626.45 \\
\hline 45 & 662.12 & 665.16 & 665.34 & 667.32 & 657.52 \\
\hline 46 & 665.74 & 669.92 & 670.00 & 676.08 & 662.39 \\
\hline 47 & 695.07 & 680.66 & 687.96 & 710.79 & 697.82 \\
\hline 48 & 717.80 & 711.29 & 693.04 & 715.30 & 712.50 \\
\hline 49 & 719.58 & 722.00 & 722.81 & 730.42 & 716.95 \\
\hline 50 & 771.81 & 734.63 & 723.35 & 753.20 & 774.08 \\
\hline 51 & 777.30 & 751.39 & 739.10 & 758.43 & 774.58 \\
\hline 52 & 802.04 & 765.50 & 776.09 & 771.82 & 785.75 \\
\hline 53 & 811.87 & 773.29 & 782.28 & 791.84 & 796.68 \\
\hline 54 & 823.95 & 790.16 & 787.46 & 798.80 & 798.92 \\
\hline 55 & 843.90 & 855.28 & 855.32 & 842.78 & 850.71 \\
\hline
\end{tabular}

TABle 1: Continued.

\begin{tabular}{lccccc}
\hline \multirow{2}{*}{ Mode } & \multicolumn{5}{c}{ Modal frequency $[\mathrm{Hz}]$ versus position } \\
& P1 & P2 & P3 & P4 & P5 \\
\hline 56 & 854.42 & 857.70 & 856.25 & 852.88 & 854.40 \\
57 & 861.35 & 860.09 & 866.13 & 855.39 & 861.48 \\
58 & 862.94 & 866.28 & 871.96 & 862.52 & 862.03 \\
59 & 872.50 & 874.73 & 884.33 & 865.95 & 875.46 \\
60 & 884.03 & 885.25 & 896.82 & 879.15 & 883.83 \\
61 & 894.59 & 889.17 & 900.29 & 901.25 & 888.56 \\
62 & 904.18 & 908.11 & 925.20 & 905.52 & 901.65 \\
63 & 907.09 & 922.27 & 944.63 & 916.35 & 903.47 \\
64 & 940.11 & 931.39 & 956.41 & 922.45 & 943.24 \\
65 & 945.23 & 933.20 & 971.06 & 931.89 & 947.01 \\
66 & 973.89 & 959.63 & 972.38 & 953.58 & 969.49 \\
67 & 981.11 & 976.12 & 980.79 & 975.14 & 972.52 \\
68 & 985.77 & 979.01 & 982.52 & 978.04 & 981.10 \\
69 & 1013.00 & 983.15 & 1002.40 & 983.45 & 1008.80 \\
70 & 1027.30 & 1013.60 & 1020.20 & 1015.80 & 1025.60 \\
71 & 1055.90 & 1031.80 & 1051.40 & 1022.00 & 1028.60 \\
72 & 1079.60 & 1054.90 & 1064.70 & 1056.40 & 1061.40 \\
73 & 1090.60 & 1084.70 & 1068.20 & 1090.80 & 1089.40 \\
74 & 1119.80 & 1090.90 & 1092.60 & 1113.80 & 1092.20 \\
75 & 1136.30 & 1095.90 & 1116.80 & 1116.20 & 1135.90 \\
76 & 1140.20 & 1141.30 & 1134.80 & 1133.80 & 1146.40 \\
77 & 1161.70 & 1153.30 & 1144.70 & 1146.70 & 1159.10 \\
78 & 1164.20 & 1177.90 & 1145.30 & 1158.50 & 1172.50 \\
79 & 1175.10 & 1196.30 & 1191.60 & 1184.80 & 1180.90 \\
80 & 1193.40 & 1220.90 & 1230.20 & 1216.10 & 1194.50 \\
\hline & & & & &
\end{tabular}

displacement of the structure is upwards and occurs at the nearest ends with respect to the positions of the platforms.

At position $\mathrm{P} 3$, the platforms are aligned with the middle point with the center axis of the LSRA base structure. Therefore, both ends are equidistant to that position, as shown in Figure 5(b). However, as the center of mass is not fully aligned with the middle point of the actuator, there is a tendency for torsional displacement, and the antisymmetric behavior is present and noticeable. Figures 11 and 12 show the modal shapes for the first four useful modes (modes 7 to 10) for comparison.

From Figures 11(b) and 12(a), respectively, it is noticeable that, for modes 8 and 9, although with identical frequency, the displacements are complementary with reference to the middle region. The mode shape in Figure 11(b) also denotes a significant displacement of some parts at the primary of the magnetic circuit, which also contributes to the modal frequency. Maintaining the middle region as a reference, one also observes that the mode shape for mode 7 (Figure 10(a)) is antisymmetric while that for mode 10 is symmetric (Figure 12(b)). Moreover, the frequency at mode 10 is almost the double for that of mode 7 which is coherent with the number of nodes (null displacement points) shown in their respective mode shapes (two and one, respectively, regarding the middle position).

Some modes correspond mainly to wave propagation through bars, along with the horizontal $(X X)$ or vertical 


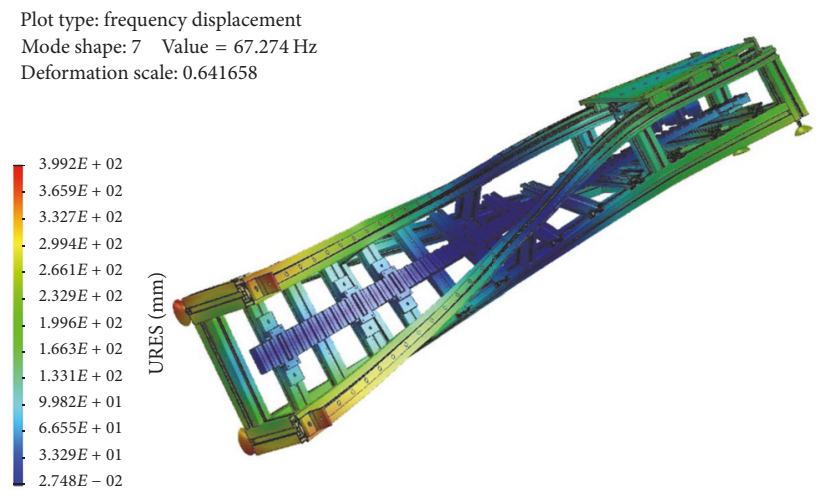

(a)

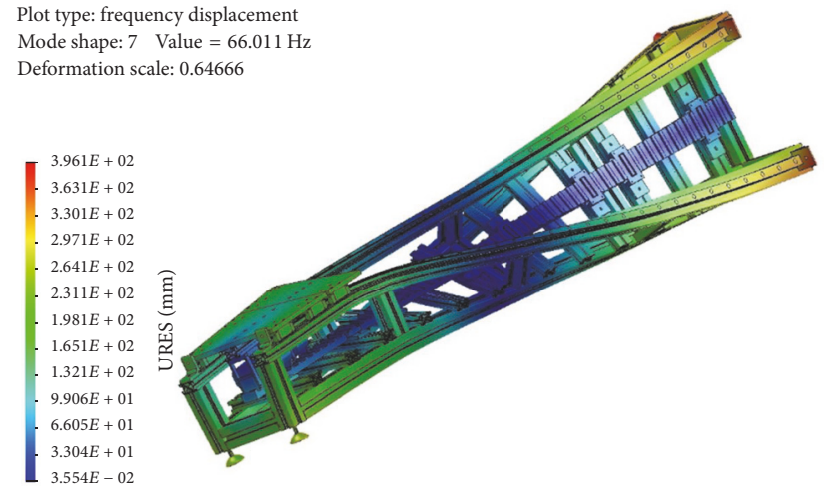

(b)

FIGURE 7: Modal shapes for mode 7 : (a) at position $\mathrm{P} 1(f \approx 67 \mathrm{~Hz}$, deformation scale $\approx 0.642)$; (b) at position $\mathrm{P} 5$ ( $f \approx 66 \mathrm{~Hz}$, deformation scale $\approx 0.646$ ).

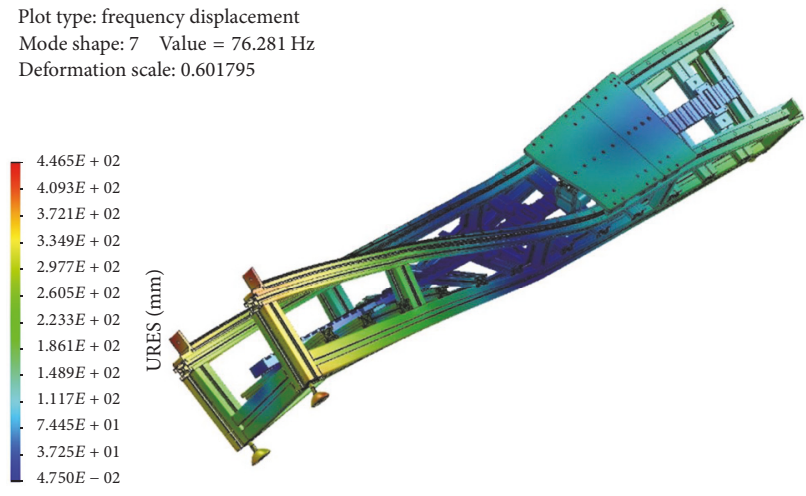

(a)

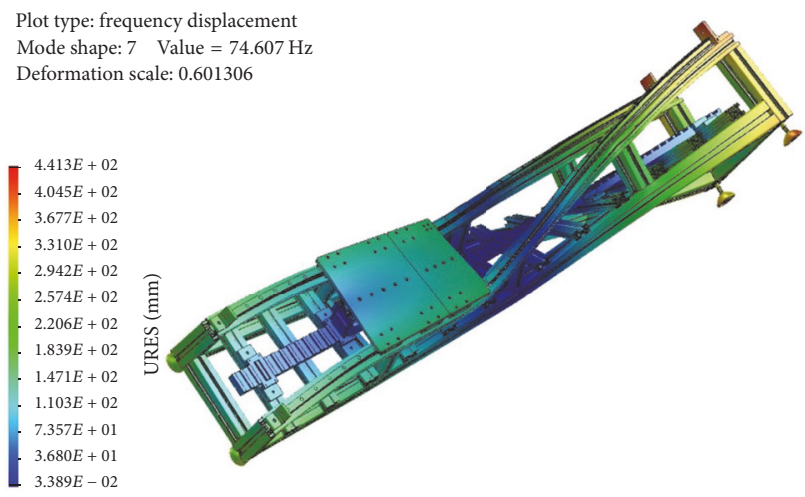

(b)

Figure 8: Modal shapes for mode 7 : (a) at position $\mathrm{P} 2(f \approx 76 \mathrm{~Hz}$, deformation scale $\approx 0.602)$; (b) at position $\mathrm{P} 4(f \approx 75 \mathrm{~Hz}$, deformation scale $\approx 0.602$ ).

\section{Plot type: frequency displacement Mode shape: 8 Value $=114.87 \mathrm{~Hz}$ Deformation scale: 0.769287}
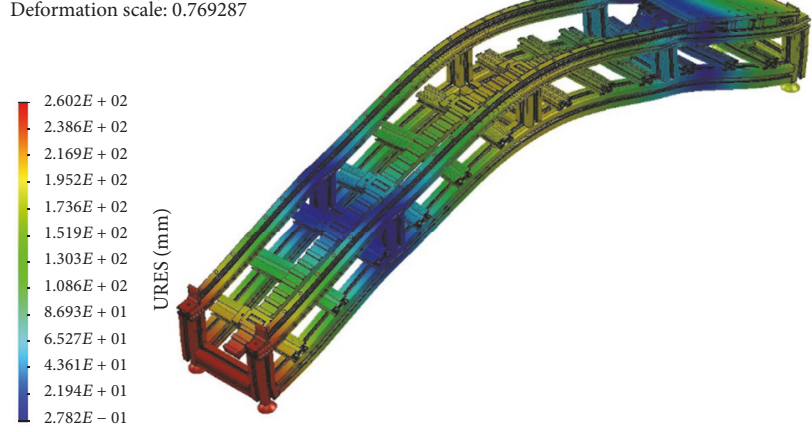

(a)
Plot type: frequency displacement Mode shape: 8 Value $=109.36 \mathrm{~Hz}$ Deformation scale: 0.794116

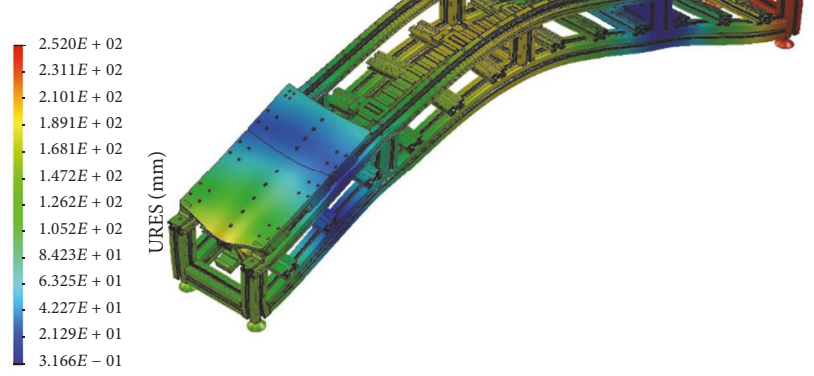

(b)

Figure 9: Modal shapes for mode 8 , with vertical displacement in both ends: (a) at position $\mathrm{P} 1(f \approx 114 \mathrm{~Hz}$, deformation scale $\approx 0.769)$; (b) for position $\mathrm{P} 5(f \approx 109 \mathrm{~Hz}$, deformation scale $\approx 0.794)$. 


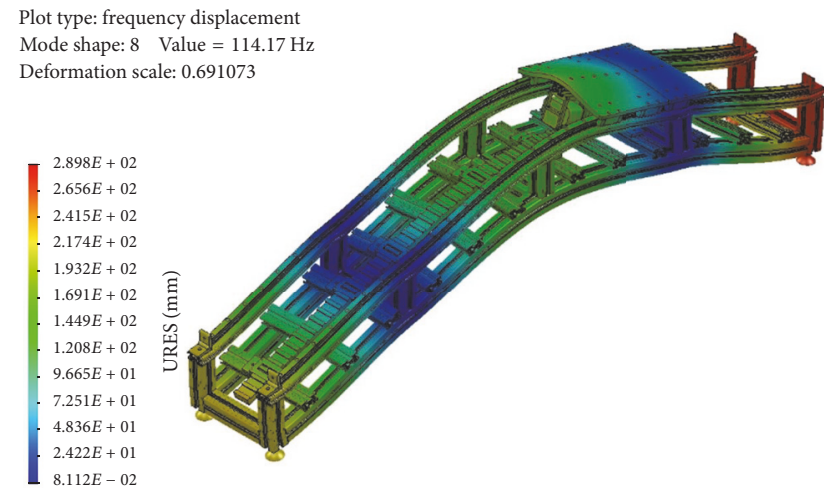

(a)

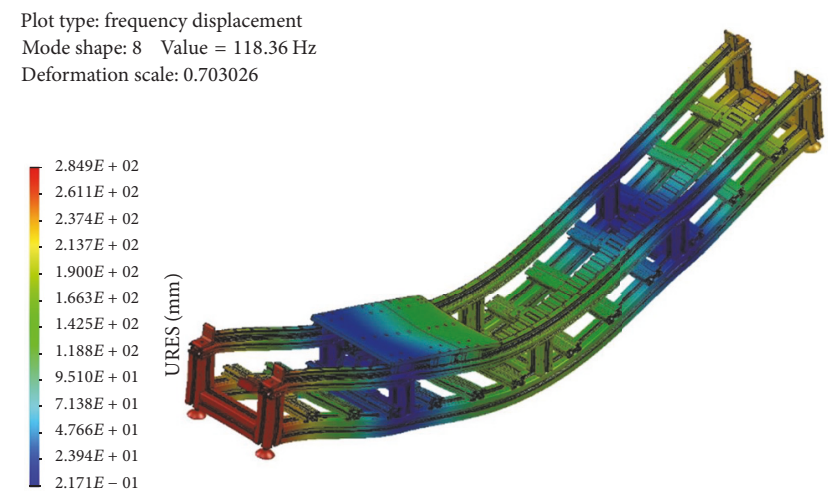

(b)

FIGURE 10: Modal shapes for mode 8 , with vertical displacement in both ends: (a) at position $\mathrm{P} 2(f \approx 114 \mathrm{~Hz}$, deformation scale $\approx 0.691$ ); (b) for position $\mathrm{P} 4(f \approx 118 \mathrm{~Hz}$, deformation scale $\approx 0.703)$.

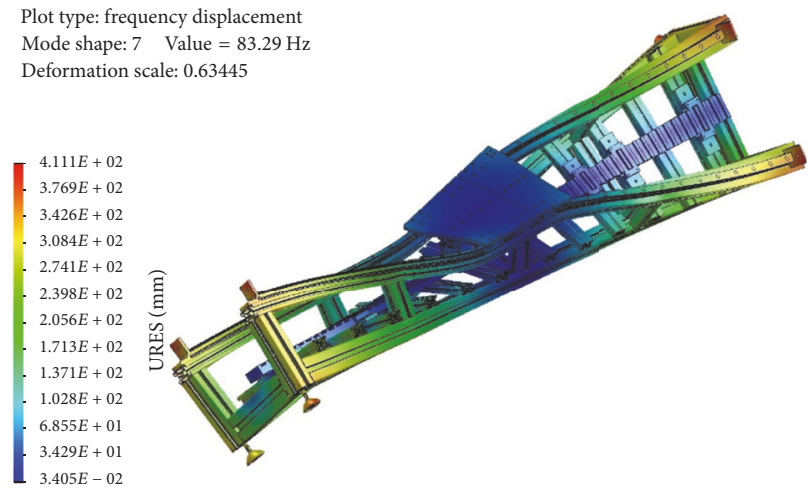

(a)
Plot type: frequency displacement
Mode shape: 8 Value $=106.91 \mathrm{~Hz}$ Deformation scale: 0.726185

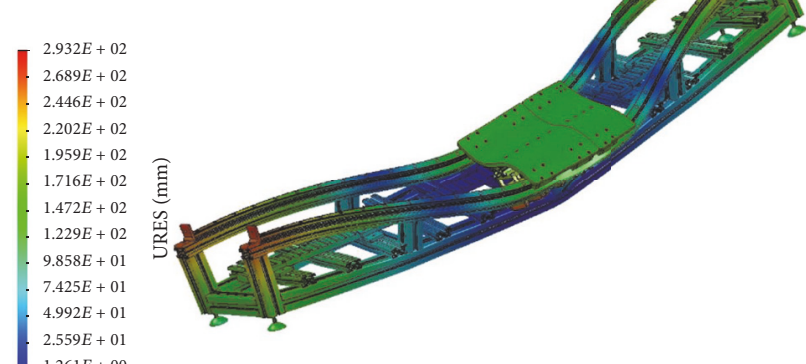

(b)

Figure 11: Modal shapes at P3 for: (a) mode 7 ( $f \approx 83 \mathrm{~Hz}$, def. scale $\approx 0.634)$, one node at the central region; (b) and for mode 8 ( $f \approx 107 \mathrm{~Hz}$, def. scale $\approx 0.602$ ), both ends move vertically.

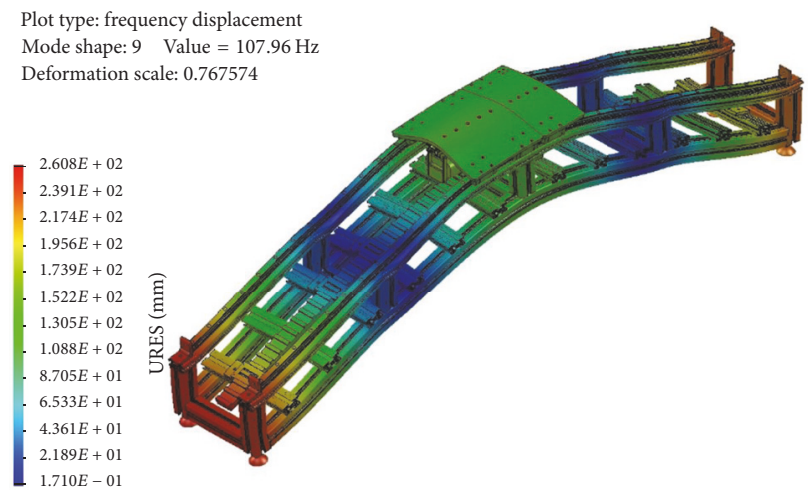

(a)

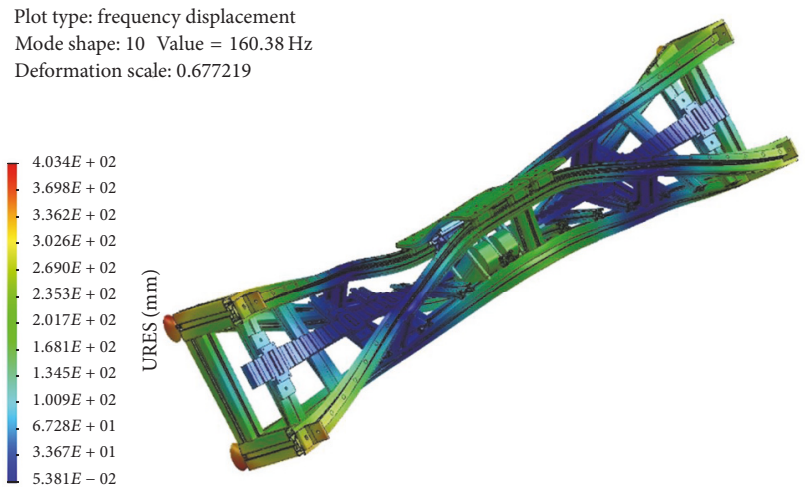

(b)

FIGURE 12: Modal shapes at P3 for: (a) mode $9(f \approx 108 \mathrm{~Hz}$, def. scale $\approx 0.767)$, one node at the central region; (b) mode $10(f \approx 161 \mathrm{~Hz}$, def. scale $\approx 0.677$ ) with two nodes almost symmetric. 
TABLE 2: Vibration modes at top longitudinal bars with transversal wave propagation.

\begin{tabular}{|c|c|c|c|c|c|c|c|c|}
\hline & \multirow{2}{*}{ Mode order } & \multicolumn{7}{|c|}{ Frequency [Hz] (mode \#) per configuration and average frequency values } \\
\hline & & P1 & P5 & $F_{\text {(av) }}[\mathrm{Hz}]$ & $\mathrm{P} 2$ & P4 & $F_{(\mathrm{av})}[\mathrm{Hz}]$ & P3 \\
\hline \multirow{7}{*}{ Bar segment $L_{L}$} & 1 & $183.65(12)$ & $182.00(11)$ & 182.83 & $201.20(13)$ & $198.15(13)$ & 199.68 & $226.10(13)$ \\
\hline & 2 & $229.72(14)$ & $226.41(15)$ & 228.07 & $265.99(17)$ & $259.09(17)$ & 262.54 & $407.88(28)$ \\
\hline & 3 & $303.34(20)$ & $291.95(20)$ & 297.65 & $459.81(32)$ & $441.77(31)$ & 450.79 & $619.41(42)$ \\
\hline & 4 & $464.62(32)$ & $452.72(31)$ & 458.67 & $639.41(44)$ & $600.96(40)$ & 620.19 & $1020.20(70)$ \\
\hline & 5 & $613.01(41)$ & $591.67(40)$ & 602.34 & $908.11(62)$ & $862.52(58)$ & 885.32 & \\
\hline & 6 & $823.95(54)$ & $798.92(54)$ & 811.44 & $1177.90(78)$ & $1158.50(78)$ & 1168.20 & \\
\hline & 7 & $1079.60(72)$ & $1028.60(71)$ & 1054.10 & & & & \\
\hline \multirow{6}{*}{ Bar segment $L_{S}$} & 1 & & & & $328.67(23)$ & $362.49(24)$ & 345.58 & $230.46(14)$ \\
\hline & 2 & & & & $665.16(45)$ & $753.20(50)$ & 709.18 & $435.21(31)$ \\
\hline & 3 & & & & & & & $670.00(46)$ \\
\hline & 4 & & & & & & & $1051.40(71)$ \\
\hline & $* 2$ & & & & $680.66(47)$ & & & \\
\hline & ${ }^{*} 4$ & & & & & & & $1064.70(72)$ \\
\hline
\end{tabular}

${ }^{*}$ Homolog modes.

$(Y Y)$ directions. Their frequencies and mode shapes can be explained via the Euler-Bernoulli theory. That is the case of the displacements related to wave propagation in horizontal and vertical directions, at the secondary and the top lateral frames. According to that theory, the bar of the secondary part can be seen as a free-free bar with multiple supports. Meanwhile, the top lateral frames are hinged-free bars whose length varies per the position of the platforms. For simplicity, we use (15) and (16) to analytically calculate the vibration frequencies corresponding to orthogonal displacements at the top lateral frames. However, the top lateral frames correspond to composite bars formed of two different parts: aluminum frame bar and a guide rail made of stainless steel (chromiumplated). Therefore, one can apply the superposition principle, considering the two sectional areas, lengths, and Young's modulus for the different materials used.

Calculating the modal frequencies for modes 9 and 11 in position $\mathrm{P} 5$ via (16) one arrives at $136.5 \mathrm{~Hz}$ and $188.2 \mathrm{~Hz}$, respectively. These analytically obtained values are close to those which resulted from FEM simulation: $137.6 \mathrm{~Hz}$ and $182 \mathrm{~Hz}$. The relative deviation of these results and those obtained analytically concerning FEM are $0.8 \%$ and $3.4 \%$, respectively. This approach has also been considered for other modes, although the resulting errors obtained with respect to the simulation results are higher. The increase in the errors is due not only to the simplicity of the discussed approach but also to the superior accuracy of the iterative method used in the simulation. Nevertheless, these examples serve to verify the consistency of the results for the simpler problems.

The modes listed in Table 2 deserve attention as they all correspond to orthogonal modes with wave propagation displacements along with the horizontal direction, that is, the $X X$ axis. All modes considered are a subset of Table 1, for which the mechanical waves propagate transversally in phase opposition, along with the subassembly formed by the guide rail and the horizontal bar at the top, on both sides of the LSRA. The number between brackets, near the frequency value, corresponds to the number of that mode as listed in Table 1. For positions P1 and P5, the platforms are aligned with one end. Because of that, the bar length, LL, is measured as the distance available to the other end of the actuator, which is identical in both cases. In positions $\mathrm{P} 2$ and P4, two different bar lengths must be considered. They correspond to the bar segments of the top bar and guide rail from the platforms with respect to the furthermost and the closest ends: the longer $\left(L_{L}\right)$ and the shorter $\left(L_{S}\right)$, respectively. At position P3, the lengths of the bars on both sides are identical. However, there are some particularities to highlight as is further referred to. The symmetric behavior of the actuator is once again evidenced for the modes listed in Table 2.

The modes and frequencies are identical for each pair of symmetric positions, namely, for positions P1 and P5 and for P2 and P4. For the latter, in particular, it also applies to the portion of the bar with a shorter length, although the differences in the frequencies are relatively higher. Moreover, as both bars lengths are identical in P3, one obtains an equal number of modes; their frequencies are also identical for same order modes.

Table 2 also shows two "singular" modes, that is, without symmetric counterpart within the range considered. Some examples are mode 47 at P2 $(680.66 \mathrm{~Hz})$ and mode 72 at P3 $(1064.70 \mathrm{~Hz})$. In spite of the difference in frequencies, these two modes are of the same order regarding the number of nodes shown at the wave that defines the mode shape. Therefore, we refer to them as homolog modes. Other examples listed for bar segment $L_{S}$, for example, are modes 45 at P2 $(665.16 \mathrm{~Hz})$ and 71 at P3 $(1051.40 \mathrm{~Hz})$, respectively. However, the displacements are towards complementary directions on their possible symmetric counterparts at bar segment $L_{L}$, for example, mode 44 at P2 $(639.41 \mathrm{~Hz})$ and mode 70 at P3 $(1020.20 \mathrm{~Hz})$, respectively. 


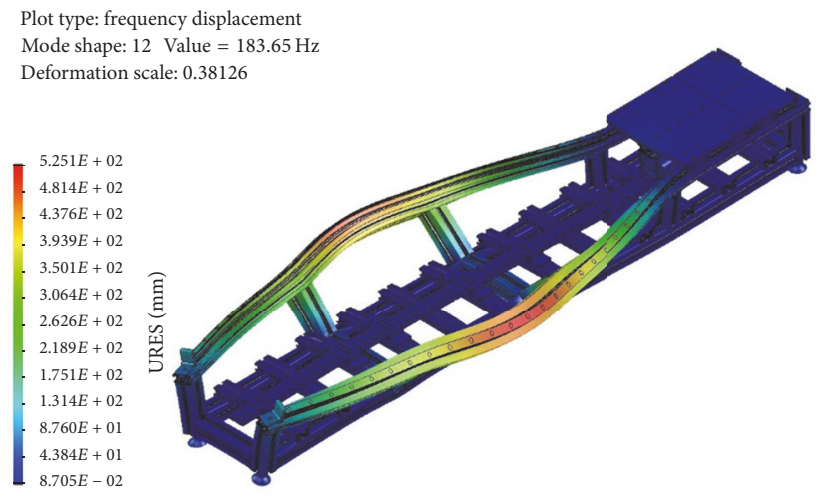

(a)

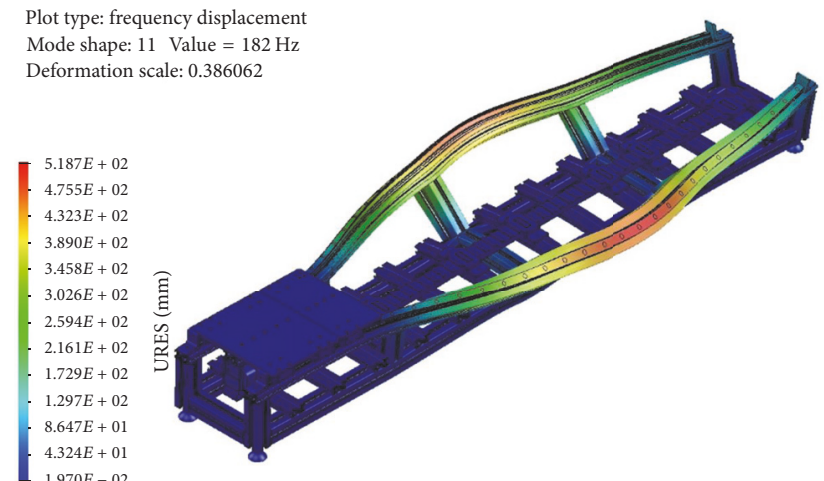

(b)

Figure 13: First-order mode shapes (mode 11), transversal displacement on top lateral bars in phase opposition: (a) position P1 ( $f \approx 184 \mathrm{~Hz}$, def. scale $=0.381)$; (b) position $\mathrm{P} 5(f \approx 182 \mathrm{~Hz}$, def. scale $\approx 0.386)$. Plot type: frequency displacement
Mode shape: 14 Value $=229.72 \mathrm{~Hz}$ Deformation scale: 0.37779

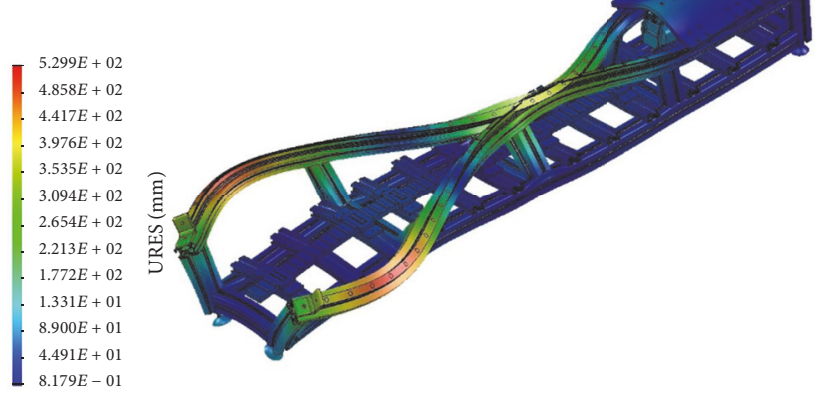

(a)

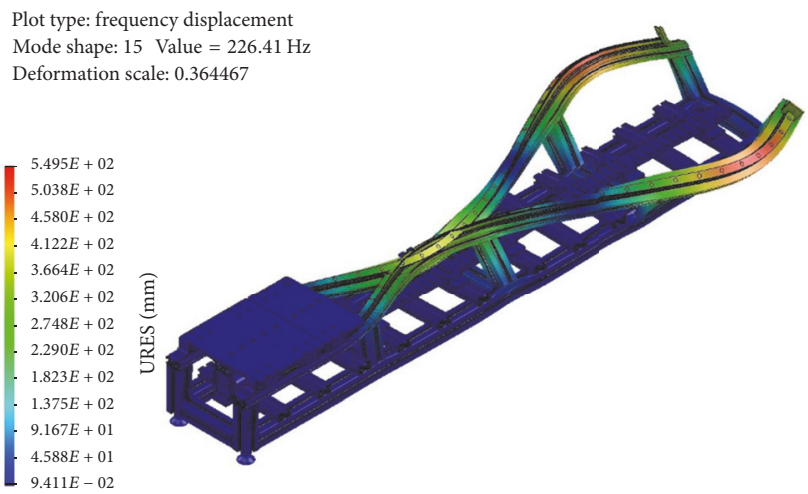

(b)

FIGURE 14: Second-order mode shapes (mode 15), with transversal displacement on top lateral bars: (a) position P1 $(f \approx 230 \mathrm{~Hz}$, def. scale $\approx$ 0.377 ); (b) position P5 ( $f \approx 226 \mathrm{~Hz}$, def. scale $\approx 0.364$ ).

All mode shapes within this set can be considered under the Euler-Bernoulli theory for simply supported hinged-free bars with free moving ends. The sequence of the modes order refers to the transversal propagation and behavior of the mechanical wave, with displacements along the horizontal direction. In a simplified approach, under the Euler-Bernoulli theory, the number of peak displacements within the length of the bar corresponds to the order of the vibration mode of the bars. The same applies to the number of nodes within that length.

Figures 13 and 14 depict the first two mode shapes related to wave propagation for positions P1 and P5. From these figures, one can see that the wave propagation causes horizontal displacements, whose modal order and mode shapes are related. The modal shape wave denotes deformations essentially at the top lateral bars, whose waves propagate in phase opposition. Similar effects occur for positions P2 and $\mathrm{P} 4$, even for higher order modes with transversal wave propagation at the top lateral bars, as shown in Figures 15 and 16.
At position P3, the lengths of the bars segments are identical on both sides, thus no meaning for shorter and longer bar segments. However, the center of mass is not aligned with the symmetry which influences the symmetric characteristic of the vibration modes, namely, the shapes of its deformation waves. The mode shapes for the first-order modes (modes 13 and 14) exhibit symmetry to the center, as shown in Figure 17, while those for the second-order modes are antisymmetric, as is observable in Figure 18. These characteristics also apply to the other modes listed in Table 2 at P3.

Note that the modal frequencies values for modes 13 and 14 in P3 are very close. Therefore, in real situations, due to imperfections and tolerances, the possibility of exciting both modes simultaneously is very high, which is not applicable to the remaining modes at position P3.

The secondary part of the magnetic circuit of the actuator is a nonuniform bar as its sectional area is not homogeneous. Nevertheless, because the teeth secondary poles are regularly and periodically distributed along the bar, its configuration 


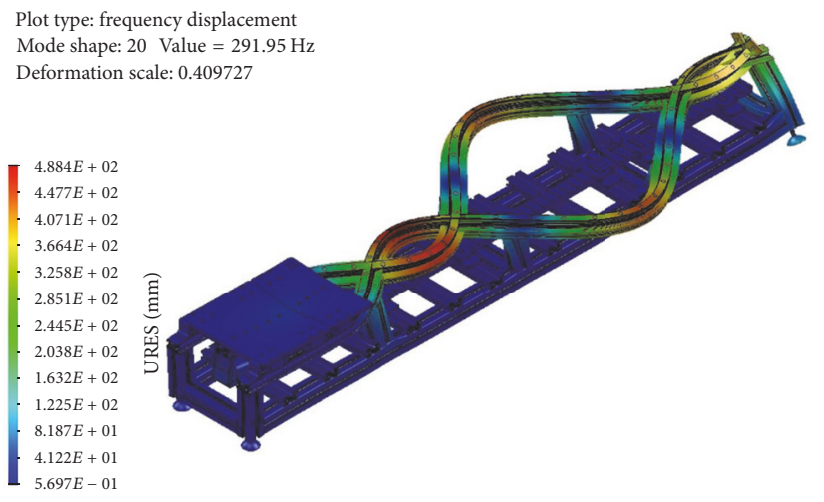

(a)

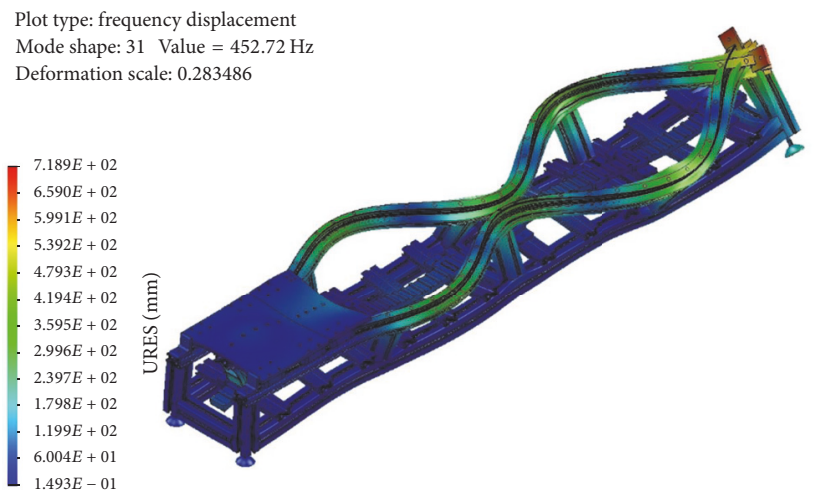

(b)

Figure 15: Mode shapes for transversal opposite waves on top lateral bars in position P5: (a) 3rd-order mode (\#20) $(f \approx 292 \mathrm{~Hz}$, def. scale = $0.409)$; (b) 4 th-order mode ( $\# 31)(f \approx 453 \mathrm{~Hz}$, def. scale $\approx 0.283)$.

Plot type: frequency displacement Mode shape: 40 Value $=591.67 \mathrm{~Hz}$ Deformation scale: 0.25908

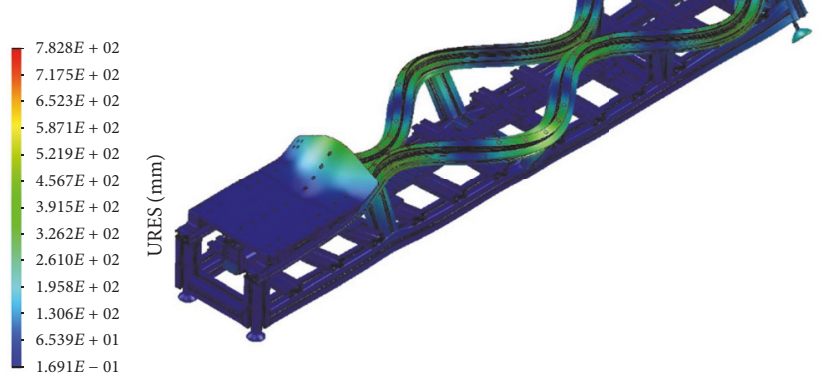

(a)
Plot type: frequency displacement Mode shape: 54 Value $=798.92 \mathrm{~Hz}$ Deformation scale: 0.307882

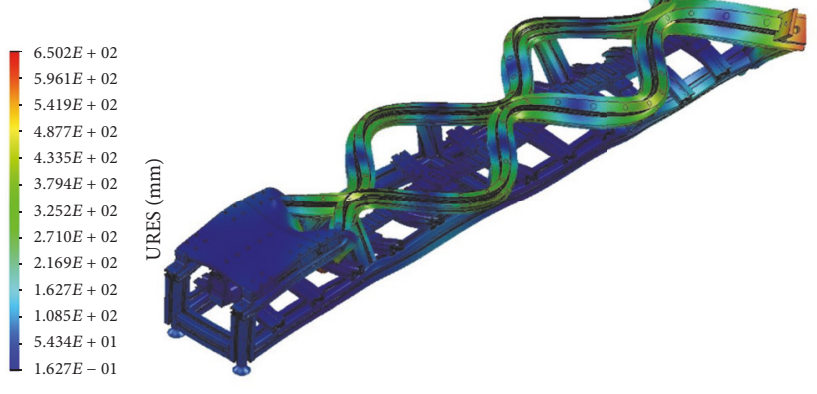

(b)

FiguRE 16: Deformation waves with transversal displacement at top lateral bars at position P5: (a) 5th-order mode $(\# 40)(f \approx 592 \mathrm{~Hz}$, def. scale $=0.259)$; (b) 6th-order mode $(\# 54)(f \approx 799 \mathrm{~Hz}$, def. scale $\approx 0.308)$.

Plot type: frequency displacement Mode shape: 13 Value $=226.1 \mathrm{~Hz}$ Deformation scale: 0.30069

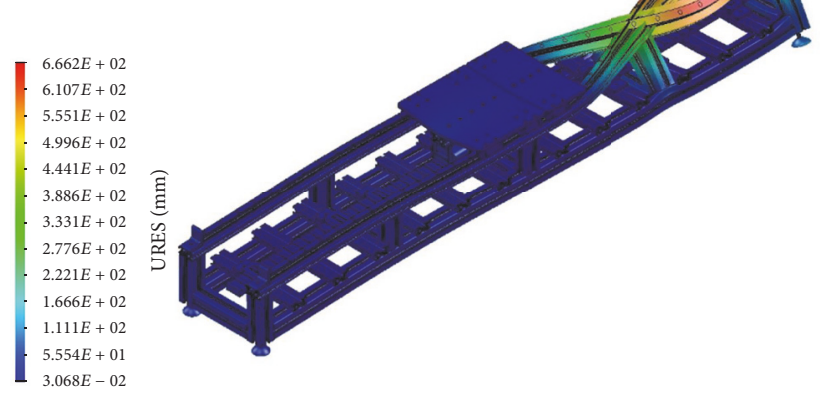

(a)

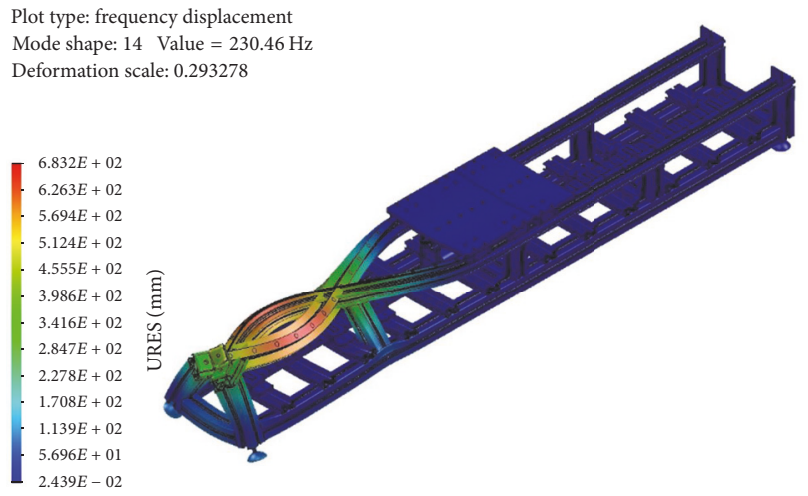

(b)

Figure 17: Mode shapes for first-order modes with transversal waves at the top lateral bars for position P3: (a) mode 13 ( $f \approx 226 \mathrm{~Hz}$, def. scale $\approx 0.301)$; (b) mode $14(f \approx 230 \mathrm{~Hz}$, def. scale $\approx 0.294)$. 
Plot type: frequency displacement Mode shape: 28 Value $=407.88 \mathrm{~Hz}$ Deformation scale: 0.264499

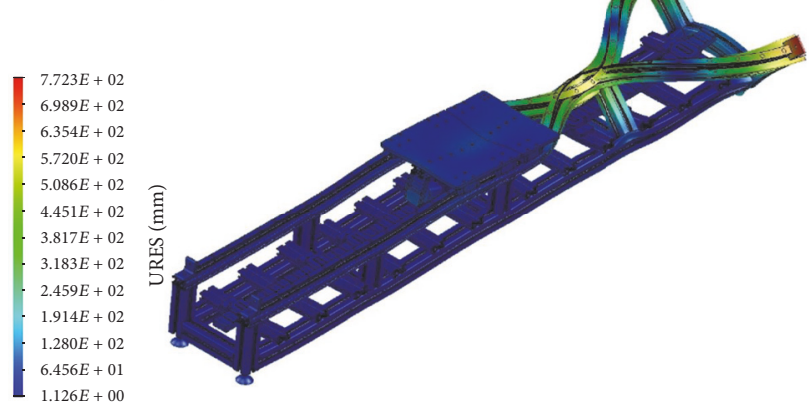

(a)

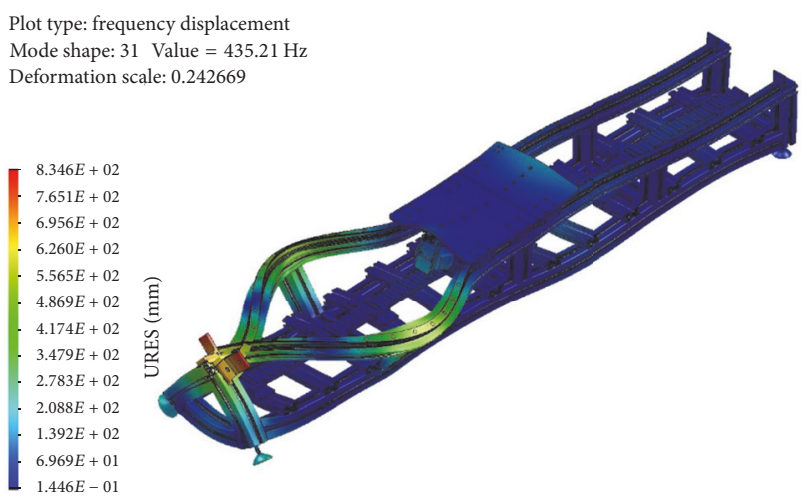

(b)

FIGURE 18: Shapes for second-order modes with transversal waves at the top lateral bars at position P3: (a) mode $28(f \approx 407 \mathrm{~Hz}$, def. scale $\approx$ 0.265 ); (b) mode 31 ( $f \approx 435 \mathrm{~Hz}$, def. scale $\approx 0.243$ ).

TABle 3: Transversal vibration modes at the bar of secondary with displacement along with vertical.

\begin{tabular}{lcccccc}
\hline \multirow{2}{*}{ Mode order } & \multicolumn{4}{c}{ Relative frequency deviation to average value per position [\%] and mode \# } \\
& $F_{\mathrm{av}}[\mathrm{Hz}]$ & $\mathrm{P} 1$ & $\mathrm{P} 2$ & $\mathrm{P} 3$ & P4 & P5 \\
\hline 9 & $\mathbf{9 7 3 . 4 0}$ & $0.05(66)$ & $0.28(67)$ & $0.11(66)$ & $0.18(67)$ & $0.40(66)$ \\
10 & $\mathbf{1 0 9 0 . 8 6}$ & $0.02(73)$ & $0.00(74)$ & $0.16(74)$ & $0.01(73)$ & $0.13(73)$ \\
\hline
\end{tabular}

denotes regularity and symmetry. Table 3 lists the 9th- and 10th-order modes related to vibrations almost exclusively with the bar of the secondary per configuration of the actuator. It also shows the relative frequency deviation to the average value of frequency for each particular order mode. The number between brackets denotes the corresponding mode per position as listed in Table 1.

The relative frequency deviation is lower than $0.4 \%$, which evidences that the vibration frequency is approximately equal for the mode order in each position. This is emphasized by the mode number which is almost the same per position and per mode order, according to the general data in Table 1. The associated mode shapes also denote consistency in the modal frequency for each position, as the differences among the frequencies are small: the deviation from the mean values. The peak displacements or the nodes visible in the mode shapes indicate the order of the mode and its relation to a free end multisupported bar.

In this case, the wave propagation generates transversal displacements along the vertical direction, that is, along the YY axis. Figures 19(a) and 19(b) illustrate the 9th-order mode for position $\mathrm{P} 2$ and the 10th-order mode at $\mathrm{P} 4$, respectively.

The parts at the primary assembly of the magnetic circuit of the LSRA are responsible for a set of vibration frequencies, for which the contributions of other parts or subassemblies are negligible. The primary subassembly is composed of three masses of copper (the coils) and S235-JR steel (the coil nucleus and teeth), which are attached to suspension to the platforms (see Figure 2(b)). The modal frequencies listed in Table 4, which are also a subset of Table 1, are substantially related to the vibration modes of the primary parts. The results are presented in a similar manner as in
Table 3: the relative frequency deviation is referenced to the average value of frequency for each particular order mode and the number between brackets denotes the corresponding mode per position as listed in Table 1 .

The values presented in Table 4 also show that the modes order and the modal frequencies are consistent with each position. No significant displacements occur for other parts or are visible from its mode shape figures, and for that reason, its representation is omitted. The differences among the frequencies for different points are small, especially for the modes of order 2 to 4 . Taking the average frequency value as a reference, we verify that, except for 1st-order mode in $\mathrm{P} 3$, the frequency deviation does not surpass $\sim 2.4 \%$, being less than $0.07 \%$ for modes of second and fourth order. The average deviation is in general less than $0.65 \%$. We also notice symmetry on the frequency values with respect to the central position of the platforms (P3), which is related to the structural characteristics of the actuator.

The complete description of all types of modes is quite a cumbersome task and that is difficult to accommodate in the paper length. Therefore, the remaining vibration modes listed in Table 1 are described briefly. Some mode shapes listed in Table 1 correspond to in-phase horizontal displacements of the top bars, for example, modes 9 and 20 in position P1 and modes 9 and 19 for position P5. Other modes correspond to in-phase or out-of-phase vertical displacements of the lateral bars. There are also vibration modes with mixed displacements in all three directions and with the torsional movement of the bars, as depicted in Figures 20(a) and 20(b).

The complete analysis of all modes is more exhaustive but difficult to accommodate in the paper length. These referred to here highlight solely some modal features with 


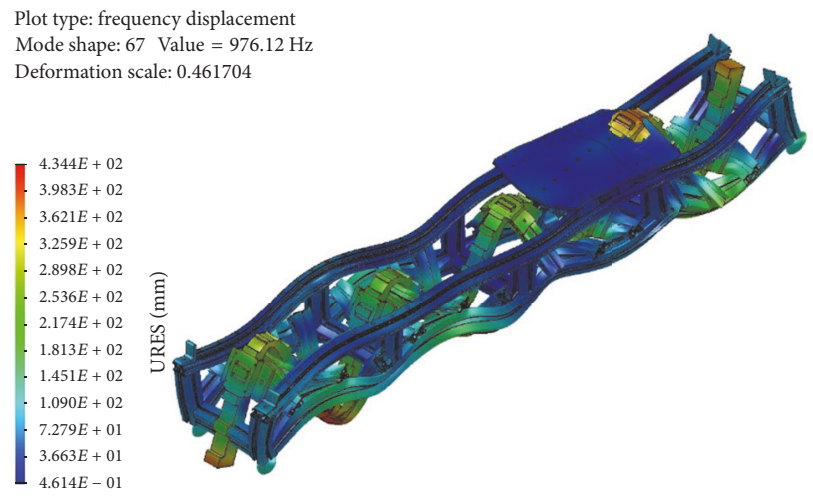

(a)

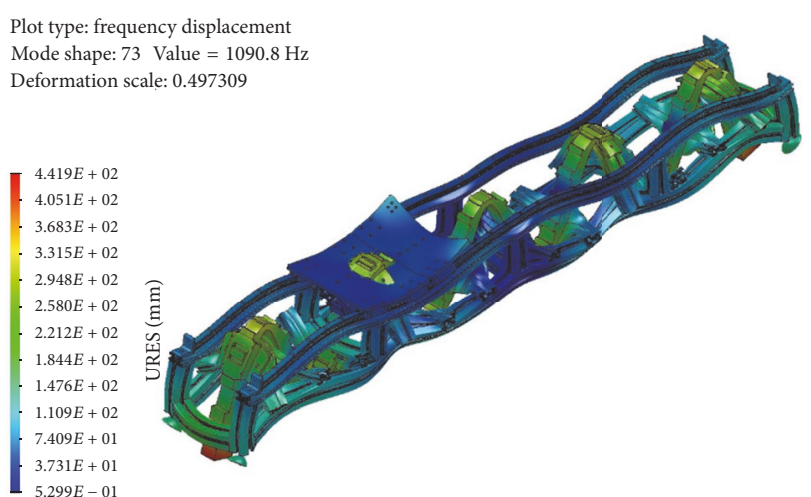

(b)

FIGURE 19: Mode shapes associated with almost exclusive vertical displacement at the bar of the secondary of magnetic circuit: (a) 9th-order mode (mode 67$)$ at position $\mathrm{P} 2(f \approx 976 \mathrm{~Hz}$, def. scale $\approx 0.462)$; (b) 10th-order mode (mode 73$)$ at position $\mathrm{P} 4(f \approx 1091 \mathrm{~Hz}$, def. scale $\approx$ 0.497).

TABLE 4: Vibration modes almost exclusively related to contributions of the primary circuit parts.

\begin{tabular}{|c|c|c|c|c|c|c|}
\hline \multirow{2}{*}{ Mode order } & \multicolumn{6}{|c|}{ Relative frequency deviation to average value per position [\%] and mode \# } \\
\hline & $F_{\mathrm{av}}[\mathrm{Hz}]$ & $\mathrm{P} 1$ & $\mathrm{P} 2$ & P3 & $\mathrm{P} 4$ & P5 \\
\hline 1 & 280.01 & $1.82(18)$ & $1.20(19)$ & $4.51(19)$ & $2.40(19)$ & $1.50(19)$ \\
\hline 2 & 393.57 & $0.05(27)$ & $0.01(27)$ & $0.06(27)$ & $0.02(28)$ & $0.02(27)$ \\
\hline 3 & 536.56 & $0.19(36)$ & $0.02(37)$ & $\begin{array}{l}0.54(36) \\
0.40(37)\end{array}$ & $0.03(37)$ & $0.20(36)$ \\
\hline 4 & 854.96 & $0.06(56)$ & $0.04(55)$ & $0.04(55)$ & $0.05(57)$ & $0.07(56)$ \\
\hline 5 & 864.67 & $0.20(58)$ & 0.19 (58) & $0.17(57)$ & 0.15 (59) & $0.30(58)$ \\
\hline 6 & 915.73 & $0.94(63)$ & $0.71(63)$ & $1.03(62)$ & $0.73(64)$ & $1.54(62)$ \\
\hline
\end{tabular}

diverse characteristics. The complete information gathered from the simulations helps to identify the most significant modal frequencies of structural origin that allow the full characterization of the LSRA regarding vibrations. The dataset and its subsequent analysis also support the validation of the vibrations of the actuator under real operation and to adopt adequate control strategies with less impact on vibrational issues.

\section{Experimental Results}

To verify the results from the simulations, we conducted experimental modal excitation tests using an electrodynamic shaker to excite the structure, collect the audio emitted, and analyze the spectral contents of these audio signals in MATLAB. The force applied by the electrodynamic shaker is sinusoidal, in a steady-state regime, with constant amplitude and the frequency varying linearly within the range from 10 to $1100 \mathrm{~Hz}$ with $10 \mathrm{~Hz}$ steps. The amplitude spectrum contents of the audio signals for positions P1, P2 are shown in Figures 21 and 22 , respectively, both obtained by using the windowed discrete Fourier transform and the Hamming window. For readability purposes, some of the most significant modal frequencies are shown and highlighted whose results correspond to approximated values.

In Figures 21 and 22 there are prominent amplitude peaks at $65 \mathrm{~Hz}$ which correspond to the first mode for P1 and P5 (dual configurations). The nearest peaks positioned at the right of these correspond to nearly $75 \mathrm{~Hz}$ and are related to the first mode in positions P2 and P4. The frequencies of 180 and $201 \mathrm{~Hz}$ correspond to modes 12 and 13, and the frequencies of 282 and $290 \mathrm{~Hz}$ are related to mode 19, all listed in Table 1. The range of frequencies near $610 / 620 \mathrm{~Hz}$ corresponds to vibrations associated with the modal frequencies of modes 40 to 44, also listed in Table 1. Notice the high concentration of frequency peaks near these values. Moreover, the highlighted frequencies, $838 \mathrm{~Hz}$ and $882 \mathrm{~Hz}$, corresponded to the modes related to vibrations almost exclusively at the parts of the primary circuit, as listed in Table 4 and discussed in the text.

The deviations of the experimental results from the values obtained with numerical simulations are explained due to the ideal contact conditions considered in the FEM. Nevertheless, the test data results are coherent and confirm those obtained from numerical methods. Thus, they can be used to support the characterization of the vibrations related to the operation of the actuator. 
Plot type: frequency displacement Mode shape: 44 Value $=645.83 \mathrm{~Hz}$ Deformation scale: 0.424894

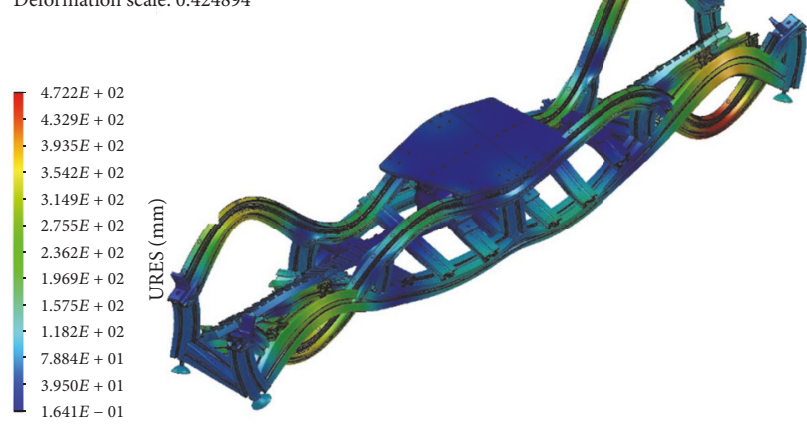

(a)

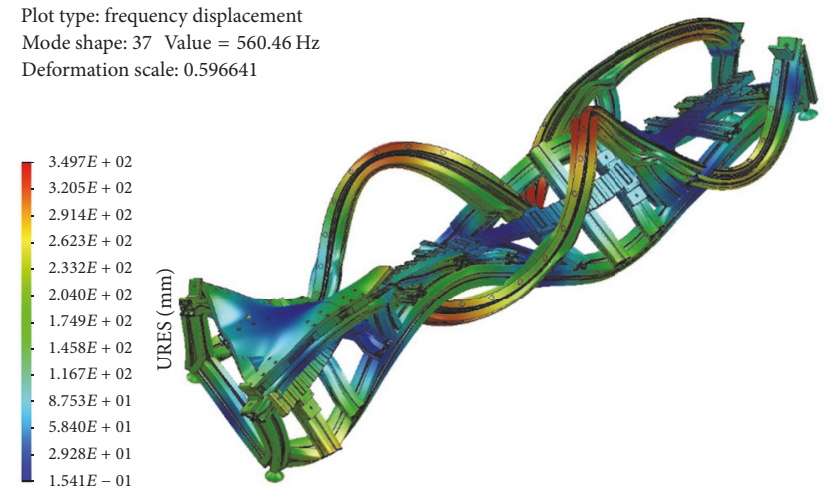

(b)

Figure 20: Modes with complex displacement function: (a) mode 44 at position $\mathrm{P} 3$ ( $f \approx 646 \mathrm{~Hz}$, def. scale $\approx 0.425$ ), multipart transversal displacements (along $Y Y$ ); (b) mode 37 for position P5 ( $f \approx 560 \mathrm{~Hz}$, def. scale $\approx 0.597$ ), transverse displacements in bars and torsion on bars and plates.

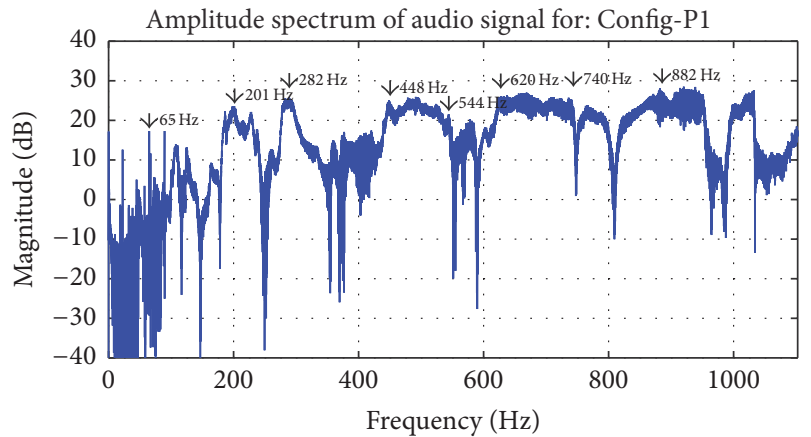

FIGURE 21: Spectral analysis showing modal frequencies excited for the LSRA at P1.

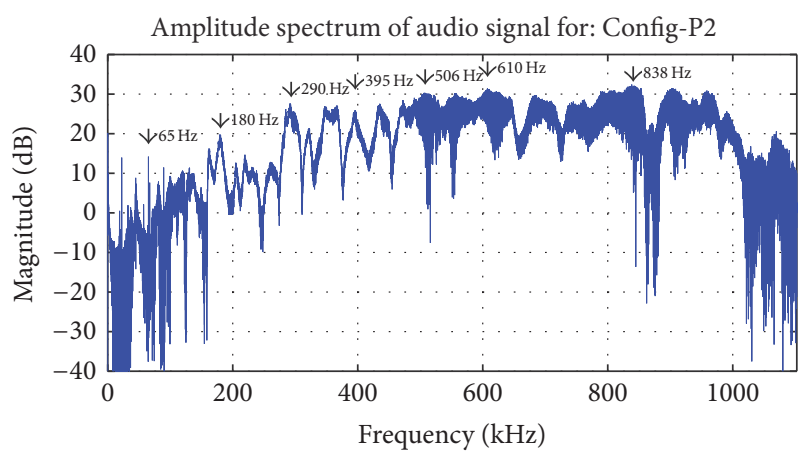

FIGURE 22: Spectral analysis showing modal frequencies excited for the LSRA at P2.

\section{Conclusions}

The analysis of the structural vibration frequencies in a threephase linear switched reluctance actuator is performed to support the characterization of the machine with respect to the excited vibrations and corresponding mode shapes. The study uses the finite element method applied to a $3 \mathrm{D}$ mechanical model and iterative methods to find the solution of the system dynamics equations. The frequency evaluation considers five different configurations of the actuator, according to the position of its platforms, and for each position, eighty modal frequencies are considered. For all considered situations, the frequency range up to nearly $1.2 \mathrm{kHz}$ contains 74 useful modal frequencies with an average variation of almost $15 \mathrm{~Hz}$ among modes. However, there are cases where the frequency difference between adjacent modes is less than $1 \mathrm{~Hz}$. The results reveal abundant modes and harmonics and the symmetry characteristics of the actuator, helping to identify the vibration modes that can be excited for different configurations of the actuator.

The mode shapes, associated with each mode, evidence the contribution of various parts of the actuator to the set of its vibration frequencies. The majority of the obtained frequencies correspond to the normal mode where the waves propagate transversally along bars or beams, in the $X X$ or $Y Y$ direction. The modes with these characteristics are associated with the lateral top bar subassemblies of the actuator structure. Each lateral subassembly is formed by a chromium-plated stainless steel guide rail mounted on the top of a rectangular aluminum profile. The waves propagate in each lateral bar subassemblies along either $X X$ or $Y Y$ direction, in phase or out of phase. However, there are other modes associated with these subassemblies, with higher complexity, in which the wave propagation implies torsional displacements. Owing to the characteristics and complexity of the structure, the mode shapes, in general, indicate wave transmission to adjacent parts and reveal their contributions to each modal frequency. On the other hand, for some special cases, the modal frequencies are associated almost solely with distinct parts or subassemblies as, for example, the primary circuit parts. For these cases, only the parts directly involved contribute to those vibration modes and the remaining parts and subassemblies do not suffer significant displacements.

The spectral analysis of the audio signals collected from experimental modal excitations serves as confirmation for 
the data obtained by the simulation and supports the characterization of the actuator. Consequently, the obtained results are consistent and well agree with the analytical estimations done for a simplified model of the system. Meanwhile, the use of FEM and iterative methods provides more accurate results. The data supplied by the simulations demonstrate the quasi-symmetric behavior of the system: that it is possible to excite similar modes at opposite positions for the actuator platforms. The overall information obtained via the FEM simulations contributes to better understanding of further experimental studies and permits one to achieve a global characterization of the actuator regarding the vibrations. The information gathered provides a possibility of modifying the structural configuration of the actuator at the design stage. It also helps to improve the control techniques for exciting the phases of the actuator, by avoiding operating regimes with frequencies near the natural vibration frequencies of the system.

\section{Conflicts of Interest}

The authors declare no conflicts of interest.

\section{Authors' Contributions}

José Salvado was responsible for adapting the 3D model of the LSRA and prepared and ran the simulations in SolidWorks. Maria do Rosário Calado supervised the work and helped to define the test configurations in conjunction with António Espírito Santo, who also supplied the initial 3D model; Anna Guerman contributed to the theoretical formulation of vibrations and helped to analyze data. All authors contributed to the writing of this research paper.

\section{Acknowledgments}

National funding resources from the Fundação para a Ciência e a Tecnologia (FCT) supported this work through the UID/EEA/50008/2013 Project.

\section{References}

[1] D. E. Cameron, J. H. Lang, and S. D. Umans, "The origin and reduction of acoustic noise in doubly salient variable-reluctance motors," IEEE Transactions on Industry Applications, vol. 28, no. 6, pp. 1250-1255, 1992.

[2] B. Fahimi, G. Suresh, and M. Ehsani, "Design considerations of switched reluctance motors: vibration and control issues," in Proceedings of the Conference Record of the 1999 IEEE Industry Applications Conference. 34th IAS Annual Meeting, vol. 4, pp. 2259-2266, 1999, Cat. No. 99CH36370.

[3] R. S. Colby, F. M. Mottier, and T. J. E. Miller, "Vibration modes and acoustic noise in a four-phase switched reluctance motor," IEEE Transactions on Industry Applications, vol. 32, no. 6, pp. 1357-1364, 1996.

[4] P. Pillay and W. Cai, "An investigation into vibration in switched reluctance motors," in Proceedings of the 33rd IAS Annual Meeting in Conference Record of IEEE Industry Applications Conference, vol. 1, pp. 341-350, 2008.
[5] W. Cai and P. Pillay, "Resonant frequencies and mode shapes of switched reluctance motors," IEEE Transactions on Energy Conversion, vol. 16, no. 1, pp. 43-48, 2001.

[6] S. A. Long, Z. Q. Zhu, and D. Howe, "Influence of load on noise and vibration of voltage and current controlled switched reluctance machines," in Proceedings of the International Conference on: Power Electronics, Machines and Drives, pp. 534-539, April 2002.

[7] Z. Tang, P. Pillay, A. M. Omekanda, C. Li, and C. Cetinkaya, "Effects of material properties on switched reluctance motor vibration determination," in Proceedings of the 38th IAS Annual Meeting on Conference Record of the Industry Applications Conference, vol. 1, pp. 235-241, October 2003.

[8] J.-P. Lecointe, R. Romary, J.-F. Brudny, and T. Czapla, "Five methods of stator natural frequency determination: case of induction and switched reluctance machines," Mechanical Systems and Signal Processing, vol. 18, no. 5, pp. 1133-1159, 2004.

[9] G. Bhuvaneswari, P. S. Rao, S. G. Thakurta, and S. S. Murthy, "Impact of dwell angle on the electromagnetic torque ripples of the switched reluctance motor," Defence Science Journal, vol. 58, no. 3, pp. 363-371, 2008.

[10] J. Li, X. Song, and Y. Cho, "Comparison of 12/8 and 6/4 switched reluctance motor: noise and vibration aspects," IEEE Transactions on Magnetics, vol. 44, no. 11, pp. 4131-4134, 2008.

[11] N. C. Lenin and R. Arumugam, "Acoustic noise, vibration, harmonics, thermal of three phase linear switched reluctance machines," in Proceedings of the 2010 Joint International Conference on Power Electronics, Drives and Energy Systems (PEDES '10), pp. 1-4, December 2010.

[12] J. Boisson, F. Louf, J. Ojeda, X. Mininger, and M. Gabsi, "Analytical approach for mechanical resonance frequencies of highspeed machines," IEEE Transactions on Industrial Electronics, vol. 61, no. 6, pp. 3081-3088, 2014.

[13] I. Husain, "Minimization of torque ripple in SRM drives," IEEE Transactions on Industrial Electronics, vol. 49, no. 1, pp. 28-39, 2002.

[14] S. A. Long, Z. Q. Zhu, and D. Howe, "Effectiveness of active noise and vibration cancellation for switched reluctance machines operating under alternative control strategies," IEEE Transactions on Energy Conversion, vol. 20, no. 4, pp. 792-801, 2005.

[15] K. A. Kasper, J. O. Fiedler, D. Schmitz, and R. W. de Doncker, "Noise reduction control strategies for switched reluctance drives," in Proceedings of the IEEE Vehicle Power and Propulsion Conference (VPPC '06), pp. 1-6, IEEE, September 2006.

[16] Y. K. Choi, H. S. Yoon, and C. S. Koh, "Pole-shape optimization of a switched-reluctance motor for torque ripple reduction," IEEE Transactions on Magnetics, vol. 43, no. 4, pp. 1797-1800, 2007.

[17] A. E. Espírito-Santo, M. R. A. Calado, and C. M. P. Cabrita, “On the influence of the pole and teeth shapes on the performance of linear switched reluctance actuator," COMPEL-The International Journal for Computation and Mathematics in Electrical and Electronic Engineering, vol. 30, no. 2, pp. 412-430, 2011.

[18] C. Lin and B. Fahimi, "Prediction of radial vibration in switched reluctance machines," IEEE Transactions on Energy Conversion, vol. 28, no. 4, pp. 1072-1081, 2013.

[19] K. Edamura and I. Miki, "Design of stator and rotor for noise reduction of SRM," in Proceedings of the 17th International Conference on Electrical Machines and Systems (ICEMS '14), pp. 1871-1874, October 2014. 
[20] C. Lin and B. Fahimi, "Prediction of acoustic noise in switched reluctance motor drives," IEEE Transactions on Energy Conversion, vol. 29, no. 1, pp. 250-258, 2014.

[21] D. W. J. Pulle, J. C. S. Lai, J. F. Milthorpe, and N. Huynh, "Quantification and analysis of acoustic noise in switched reluctance drives," in Proceedings of the 5th European Conference on Power Electronics and Applications, vol. 6, pp. 65-70, IEEE, Brighton, UK, September 1993.

[22] P. Vijayraghavan and R. Krishnan, "Noise in electric machines: a review," IEEE Transactions on Industry Applications, vol. 35, no. 5, pp. 1007-1013, 1999.

[23] J. O. Fiedler, K. A. Kasper, and R. W. De Doncker, "Acoustic noise in switched reluctance drives: an aerodynamic problem?" in Proceedings of the IEEE International Conference on Electric Machines and Drives, pp. 1275-1280, May 2005.

[24] A. E. Santo, M. D. R. Calado, and C. Cabrita, "Static simulation of a linear switched reluctance actuator with the flux tube method," Advances in Electrical and Computer Engineering, vol. 10, no. 2, pp. 35-42, 2010.

[25] X. Xue, K.-W. E. Cheng, Z. Zhang, J. Lin, and N. Cheung, "A novel method to minimize force ripple of multimodular linear switched reluctance actuators/motors," IEEE Transactions on Magnetics, vol. 48, no. 11, pp. 3859-3862, 2012.

[26] J. Salvado, A. E. Santo, and M. Do Rosário Calado, "Vibration analysis of a linear switched reluctance actuator," in Proceedings of the International Conference on Computer as a Tool (EUROCON '11), pp. 1-4, April 2011.

[27] Z. Q. Zhu, B. Lee, L. Huang, and W. Chu, "Contribution of current harmonics to average torque and torque ripple in switched reluctance machines," IEEE Transactions on Magnetics, vol. 53, no. 3, pp. 1-9, 2017.

[28] B. Ganji and M. H. Askari, "Analysis and modeling of different topologies for linear switched reluctance motor using finite element method," Alexandria Engineering Journal, vol. 55, no. 3, pp. 2531-2538, 2016.

[29] R. Krishnan, Switched Reluctance Motor Drives: Modeling, Simulation, Analysis, Design, and Applications, CRC Press, Boca Raton, Fla, USA, 2001.

[30] J. Jacques, "Noise and standardization, focusing on machinery and workplace domains," in Proceedings of the Joint BalticNordic Acosutics Meeting, pp. 8-10, June 2001.

[31] L. Meirovitch, Fundamentals of Vibrations, McGraw-Hill, Boston, Mass, USA, 2001.

[32] A. G. Piersol, T. L. Paez, and C. M. Harris, Harris' Shock and Vibration Handbook, McGraw-Hill, New York, NY, USA, 6th edition, 2010.

[33] A. E. Santo, R. Calado, and C. M. Cabrita, "Sliding mode position controller for a linear switched reluctance actuator," in Sliding Mode Control, A. Bartoszewicz, Ed., pp. 181-202, In Tech Open, Rijeka, Croatia, 2011.

[34] P. J. P. Gonçalves, M. J. Brennan, and S. J. Elliott, "Numerical evaluation of high-order modes of vibration in uniform EulerBernoulli beams," Journal of Sound and Vibration, vol. 301, no. 3-5, pp. 1035-1039, 2007.

[35] S. A. Hambric, "Structural acoustics tutorial-Part 1: vibrations in structures," Acoustics Today, vol. 2, no. 4, pp. 21-33, 2006.

[36] A. W. Leissa, Vibration of Plates, National Aeronautics and Space Administration, 1st edition, 1969.

[37] B. Schwarz and M. Richardson, "Proportional damping from experimental data," in Proceedings of the 31st International Modal Analysis Conference on Structural Dynamics (IMAC '13), pp. 179-186, February 2013. 


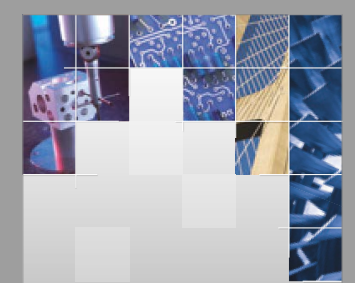

\section{Enfincering}
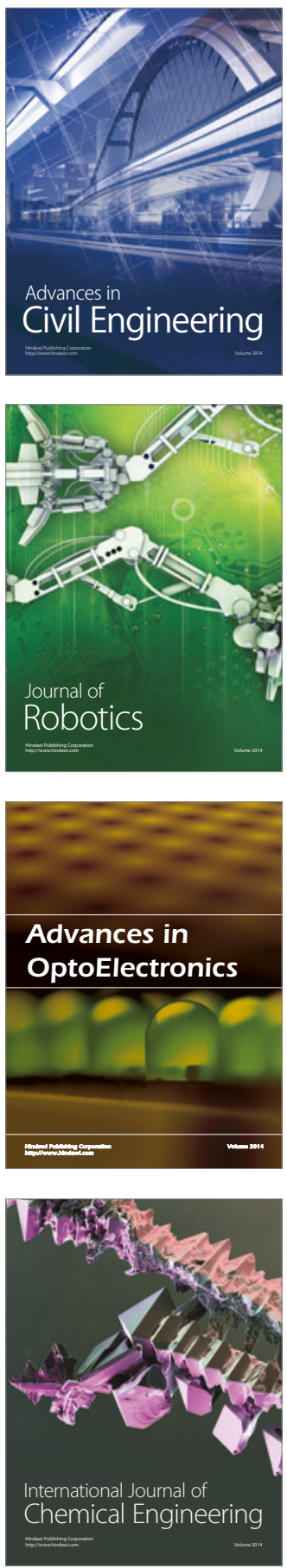

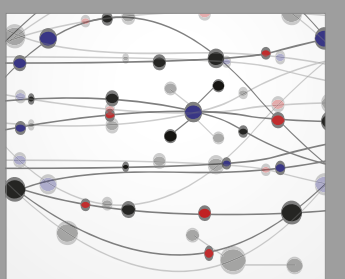

The Scientific World Journal

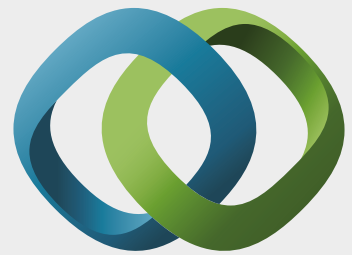

\section{Hindawi}

Submit your manuscripts at

https://www.hindawi.com
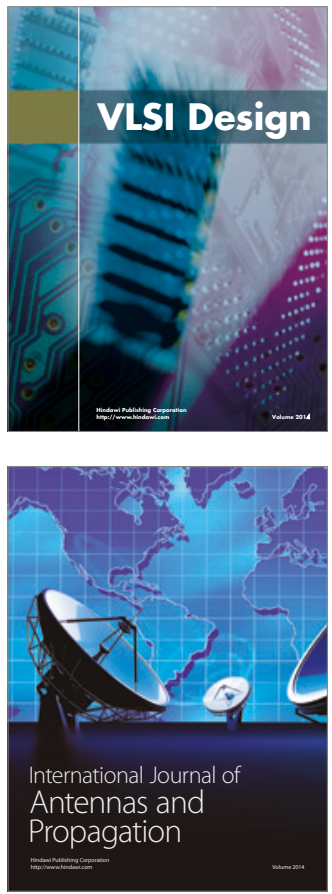

\section{Rotating}

Machinery
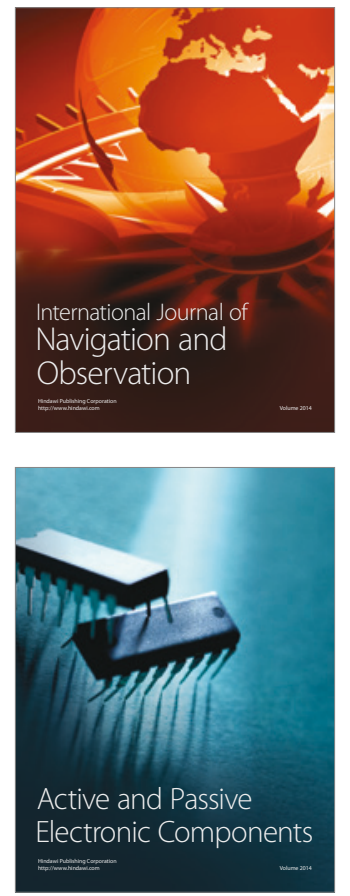
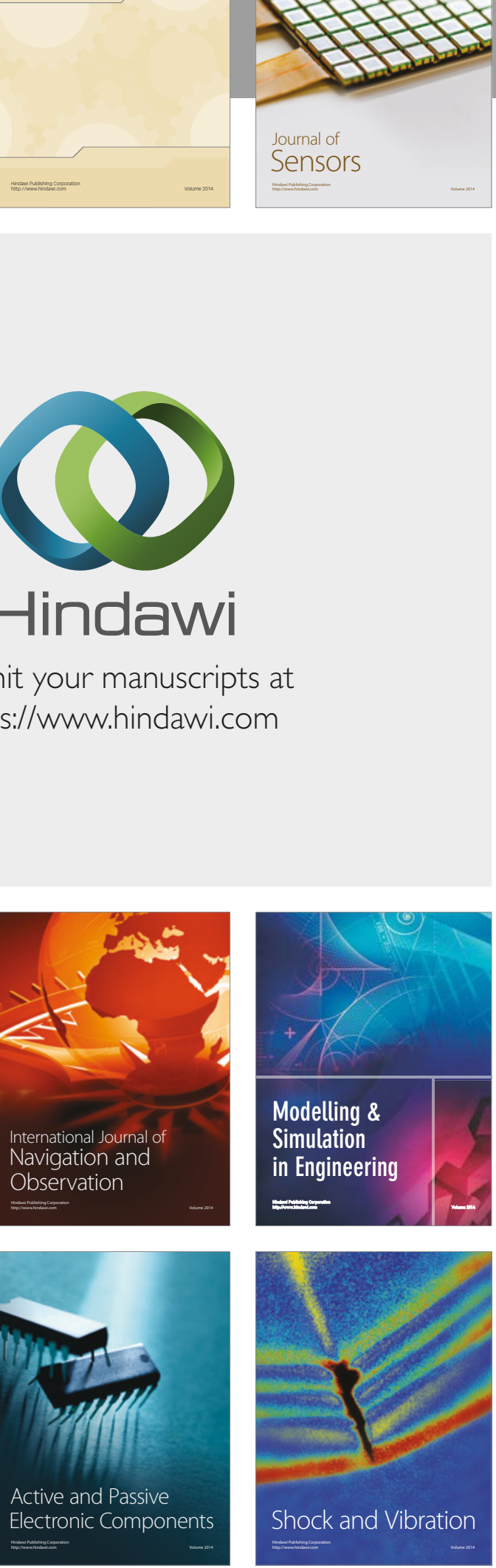
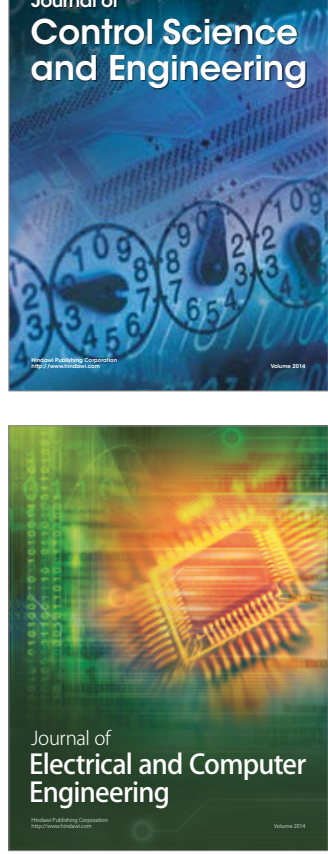

Distributed

Journal of

Control Science

and Engineering
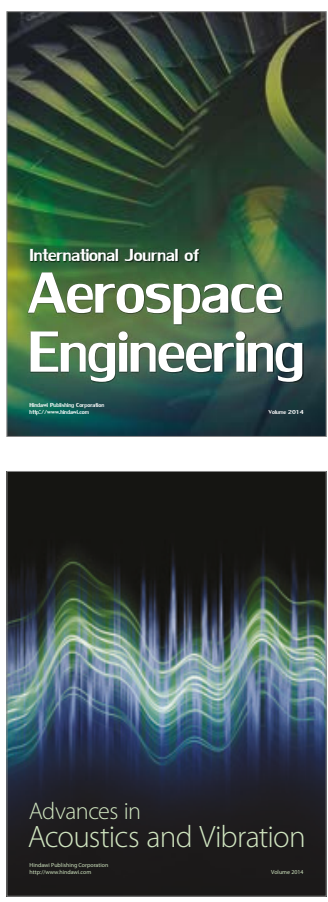

Sensor Networks 